\title{
Identification of sex determination genes and their evolution in Phlebotominae sand flies (Diptera, Nematocera)
}

\author{
Valeria Petrella', Serena Aceto ${ }^{1}$, Vincenza Colonna ${ }^{2}$, Giuseppe Saccone ${ }^{1}$, Remo Sanges ${ }^{3,4}$, Nikola Polanska ${ }^{5}$ \\ Petr Volf ${ }^{5}$, Luigi Gradoni ${ }^{6}$, Gioia Bongiorno ${ }^{6}$ and Marco Salvemini ${ }^{1 *}$ (iD
}

\begin{abstract}
Background: Phlebotomine sand flies (Diptera, Nematocera) are important vectors of several pathogens, including Leishmania parasites, causing serious diseases of humans and dogs. Despite their importance as disease vectors, most aspects of sand fly biology remain unknown including the molecular basis of their reproduction and sex determination, aspects also relevant for the development of novel vector control strategies.

Results: Using comparative genomics/transcriptomics data mining and transcriptional profiling, we identified the sex determining genes in phlebotomine sand flies and proposed the first model for the sex determination cascade of these insects. For all the genes identified, we produced manually curated gene models, developmental gene expression profile and performed evolutionary molecular analysis. We identified and characterized, for the first time in a Nematocera species, the transformer (tra) homolog which exhibits both conserved and novel features. The analysis of the tra locus in sand flies and its expression pattern suggest that this gene is able to autoregulate its own splicing, as observed in the fruit fly Ceratitis capitata and several other insect species.

Conclusions: Our results permit to fill the gap about sex determination in sand flies, contribute to a better understanding of this developmental pathway in Nematocera and open the way for the identification of sex determining orthologs in other species of this important Diptera sub-order. Furthermore, the sex determination genes identified in our work also provide the opportunity of future biotechnological applications to control natural population of sand flies, reducing their impact on public health.
\end{abstract}

Keywords: Sex determination, Sand fly, Nematocera, Genomic data mining, Alternative splicing, Transformer

\section{Background}

In animals, sex determination is the process by which early embryos of metazoan species with sexual reproduction operate a binary decision between two conditions: male or female development. This key decision results in individuals that can be identified as males, females, or in some cases hermaphrodites and, in species with a genetic sex determination system, underlies genomic differences between sexes. In most cases, the presence of heteromorphic sexual chromosomes represents the primary signal for sex determination. According to the initial decision, the primary signal is then transduced,

\footnotetext{
* Correspondence: marco.salvemini@unina.it

1'Department of Biology, University of Naples Federico II, Naples, Italy

Full list of author information is available at the end of the article
}

through a genetic pathway organized in a cascade of regulatory genes, to downstream regulators responsible for sexual differentiation [1-3].

Insects are among the largest taxonomic animal groups on Earth and, not surprisingly, they exhibit a wide variety of sex determining systems, with highly variable primary signals and widely conserved genetic transduction mechanisms to downstream regulators [4-6]. Drosophila melanogaster (Diptera, Drosophilidae) is the model species where sex determination is known at the higher level of molecular resolution (Fig. 1). In this species, sex determination is controlled by five main genes, Sex-lethal $(S x l)$, transformer (tra), transformer-2 (tra-2), doublesex ( $d s x$ ) and fruitless (fru), hierarchically organized in a regulative cascade: $S x l->$ tra $+\operatorname{tra}-2->d s x$, fru. This cascade is 


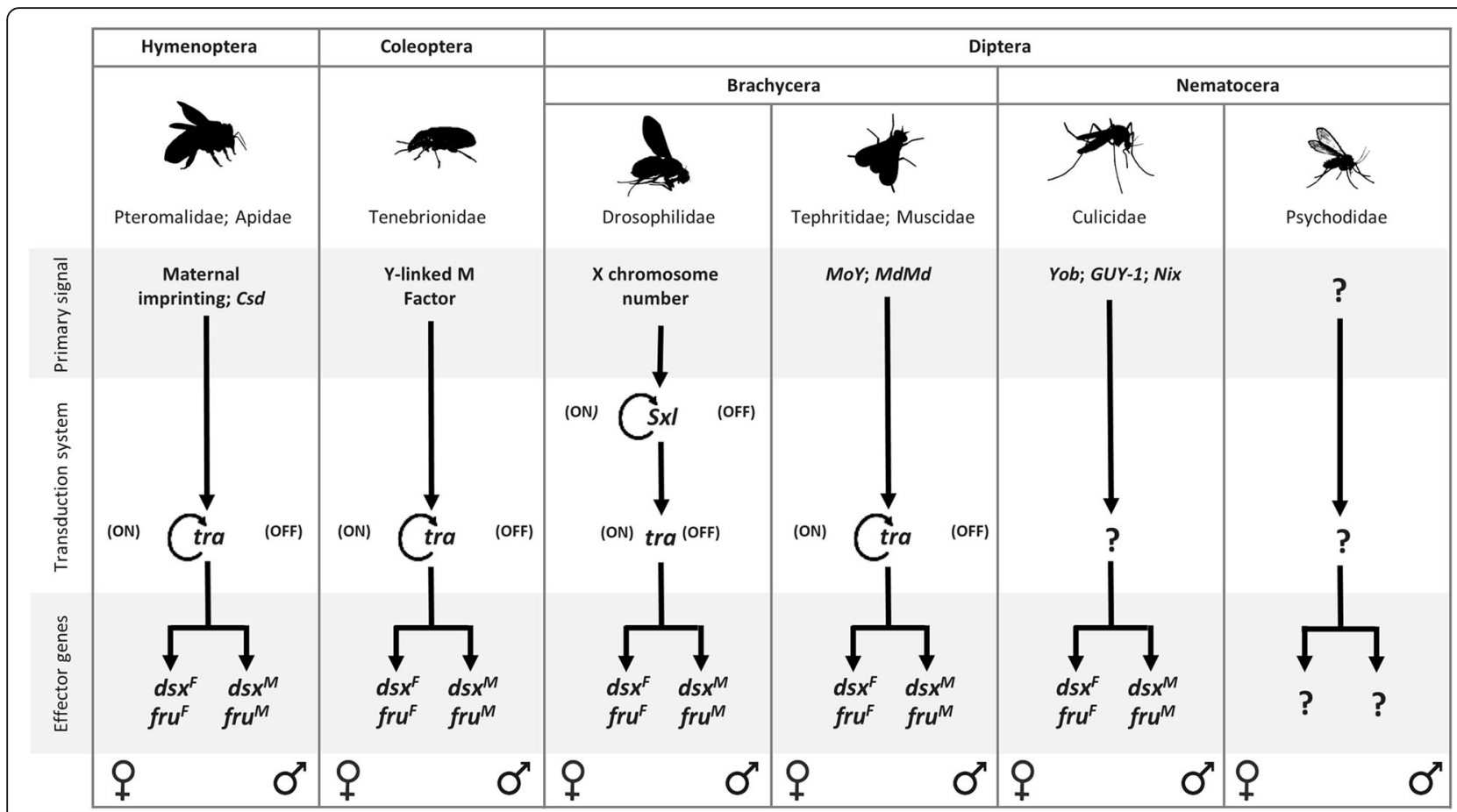

Fig. 1 Sex determination in insect species. Orders, suborders and families of species with identified sex determining genes reported in figure are indicated. In the model system Drosophila melanogaster, the presence of two X chromosomes in the female embryo activates the Sex-lethal gene $(S x I)$ which, acting as a gene-specific splicing regulator, promotes the female-specific splicing of its own pre-mRNA and of the pre-mRNA of the downstream gene transformer (tra) in the regulative cascade. Tra and the non-sex-specific auxiliary factor transformer-2 (tra-2) encode for splicing factors (TRA and TRA-2 proteins) able to control the splicing of at least two downstream target genes, responsible of sexual differentiation and courtship behaviour: doublesex ( $d s x)$ and fruitless (fru), respectively. Both genes encode for sex-specific transcription factors that potentially binds to multiple genome loci, leading to sex-specific gene expression and subsequent sexual differentiation. In male embryo, the absence of the functional SXL protein leads to the male-specific splicing of tra, $d s x$ and fru pre-mRNAs resulting in the activation of the male development program. In Hymenoptera, Coleoptera, and Diptera (Brachycera), different primary signals set the activity state of the tra homolog able to autoregulate its own splicing in the female sex and to determine female development. In mosquitoes (Diptera, Nematocera) $d s x$ and fru genes exhibit, as for Brachycera species, a conserved alternative splicing regulation, producing sex-specific protein isoforms. Recently, genomic/ transcriptomic studies of sex determination led to the discovery of novel primary signals including the Y-linked genes Yob and Guy-1 in the malaria vectors Anopheles gambiae and An. stephensi, respectively, and the putative splicing factors Nix in the dengue vector Aedes aegypti. These primary signals are supposed to act upstream of $d s x$ and fru genes in the sex determination cascade. However, their mechanism of action, direct or indirect, and the possible presence of an intermediate upstream regulator of $d s x$ and fru splicing, is still an open question

activated by a primary signal represented by the number of $\mathrm{X}$ chromosomes $[7,8]$. In the last 20 years, homologybased approaches in species belonging to various insects orders (Diptera, Coleoptera, Lepidoptera, Hymenoptera) led to discover only partial conservation of the Drosophila sex determination genetic pathway: in all species studied the $S x l$ ortholog was not involved in sex determination while the tra ortholog is able to control the femalesplicing of its own pre-mRNA as well as to control, similarly to Drosophila, the female-specific splicing of the $d s x$ and $f r u$ downstream genes $[6,9,10]$. In female embryos, the maternal tra contribution establishes the female-specific autoregulatory splicing of tra and leads to female development, which is epigenetically maintained during development in the absence of the initial positive signal. In male embryos, the establishment of tra autoregulatory feedback loop is impaired by the presence of a masculinizing factor able to interfere with the maternal and/or the zygotic tra function, blocking its positive autoregulation and leading to male development, as shown recently in Musca domestica [11]. The molecular mechanism underlying the autoregulation of the tra gene in insects is still unknown.

The tra + tra2- $>d s x /$ fru sex determination module with an autoregulating tra, firstly discovered in the Mediterranean fruit fly Ceratitis capitata [12, 13], represents the core pathway of insect sex determination [14]. The only remarkable exception is represented by the Lepidoptera order, where a different sex determination system exists with the primary signal constituted by a small RNA, the absence of the tra ortholog and the $d s x$ splicing controlled by different splicing regulators [15]. 
Within Diptera, the insect order where sex determination has been studied in the largest number of species, the tra ortholog has been identified only in species belonging to the Brachycera suborder [16-22]. For the basal suborder Nematocera, which includes very important hematophagous vector species such as mosquitoes, sand flies and black flies, the tra ortholog or its functional analog has not yet been found in any species and limited knowledge is available in general about sex determination, mainly restricted to mosquito species [2330] (Fig. 1).

Within Nematocera, phlebotomine sand flies are second only to mosquitoes in importance as a vector of pathogens that cause diseases to humans and animals worldwide, including leishmaniases, sand fly fever, bartonellosis, vesicular stomatitis, encephalitis and meningitis [31]. Among the over 800 species of sand fly described to date, 98 are proven or suspected vectors of human leishmaniases; these include 42 Phlebotomus species in the Old World and 56 Lutzomyia species (sensu) in the New World [32]. Leishmaniasis are diseases of great public health concern, being endemic in over 98 countries, with more than 350 million people at risk and 2, 357,000 disability-adjusted life years lost [33]. It is estimated that about 1.3 million new cases of leishmaniasis (0.2-0.4 million visceral and 0.7-1.2 million cutaneous leishmaniasis) occur every year, with 20,000-40,000 deaths caused by the visceral form. With expanding endemicity, leishmaniasis is becoming a worldwide reemerging public health problem [34].

Despite their importance as disease vectors, most aspects of sand fly biology remain unknown, including sex determination and sexual differentiation. To fill this gap and contribute to a better understanding of the evolution of sex determination mechanisms in insects, in the present study we applied a genomic/transcriptomic data mining approach to identify and molecularly characterize sex determining genes in sand fly species. For the first time we present in a unique study the analysis of the key components of the sex determining cascade, also identifying the first transformer homolog in a Nematocera species.

\section{Results and discussion}

Identification of PpeSxl, Ppetra, Ppetra-2, Ppedsx, and Ppefru sex determining genes in the sand fly Phlebotomus perniciosus

In the Old World, the sand fly Phlebotomus perniciosus (Diptera, Nematocera) is the main vector of Leishmania infantum (Kinetoplastida: Trypanosomatidae), the parasitic protozoan that causes visceral and cutaneous leishmaniasis in humans and canine reservoir host, as well as of various known and emerging arboviruses considered relevant from an European public health perspective
(Toscana Virus, Naples Virus, Sicilian Virus) [35]. Proteins encoded by insect sex determining genes are characterized by domains very well conserved across insect orders and distinctive of each gene family: the DNAbinding DM (Doublesex/Mab3) domain for the DSX proteins [36], the protein-protein BTB (Broad-Complex, Tramtrack and Bric a brac) binding domain for the FRU proteins [37] and the RNA-binding RRM (RNA Recogni-

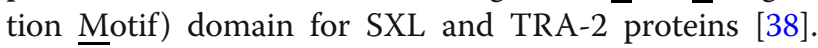
Conversely, the female-specific serine-arginine rich TRA is a fast-evolving protein which exhibits general low conservation of its primary sequence, even between closely related species, and absence of functional characterized domains. In most of the insect species analyzed to date, the only conserved parts of TRA are the TRACAM (Ceratitis-Apis-Musca), the DIPTERA and the HYMENOPTERA domains, all with unknown function [14, 19, 39]. The TRACAM domain has been proposed to be involved in the autoregulatory activity of the tra gene; however, functional molecular or biochemical data supporting this hypothesis are not available to date $[4,14]$.

We performed a TBLASTN search against the available P. perniciosus adult transcriptome database (http:// pernibase.evosexdevo.eu) [40] to identify transcripts encoding for sex determining proteins, using other insects sex determining protein sequences as query terms (Additional file 1: Table S1). In addition, we searched by TBLASTN the orthologs of the recently identified primary signals of mosquito sex determination, the $\mathrm{Yob}$ (Anopheles gambiae), Guy1 (An. stephensi) and Nix (Aedes aegypti) genes. We did not identify any ortholog of these genes in P. perniciosus [27-29].

In P. perniciosus we identified the complete open reading frames (ORF) of the transcripts encoding for the putative SXL, TRA-2 and male- and female-specific isoforms of DSX (Additional file 2: Figures S1-S5). We named the corresponding genes as PpeSxl, Ppetra-2, and Ppedsx. In addition, we identified partial ORFs encoding for FRU, and we named the gene as Ppefru. The incomplete transcripts encoding for FRU proteins lack their 3' ends and therefore complete ORFs were obtained by 3' RACE (Additional file 2: Figures S6-S7), as described in Supplementary Methods (Additional file 15). Using the TBLASTN approach, no tra ortholog was found in the $P$. perniciosus transcriptome. This result was expected, due to the low level of nucleotide and protein sequence conservation of the tra gene, also among closely related insect species and considering that the cloning of tra in Ceratitis was performed by synteny rather than by sequence similarity $[16,17,41,42]$.

We validated the transcription and the splicing pattern of PpeSxl, Ppetra-2, Ppedsx and Ppefru by RT-PCR on mRNAs extracted from adult $P$. perniciosus males and females, using the Ppesod gene as endogenous positive 
control and to exclude genomic DNA contamination of the cDNAs (Fig. 2a). Primer pairs for PpeSxl and Ppetra2 span the region upstream the translation start site and downstream the stop codon. Primer pairs for Ppedsx and Ppefru span the region that includes the sex-specific alternative splicing sites.

The RT-PCR primer pairs for the PpeSxl transcript amplified in both sexes multiple non-sex-specific transcripts, probably produced by alternative splicing (Additional file 2: Figure S8), as observed in other insect species [43, 44]. Functional analyses of $S x l$ in several dipteran species $[45,46]$ show that $S x l$ is a master switch gene of sex determination only in Drosophilidae $[9,47]$. Therefore, we believe that $S x l$ is probably not essential for the sex determination in $P$. perniciosus and decided to exclude it from further analyses.

The RT-PCR analysis of the Ppetra-2 transcript showed a non-sex-specific expression at adult stage and revealed the existence of a second isoform (Ppetra-2B) expressed in both sexes (Fig. 2b). Cloning and sequencing of Ppetra-

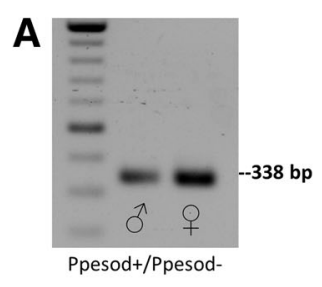

B

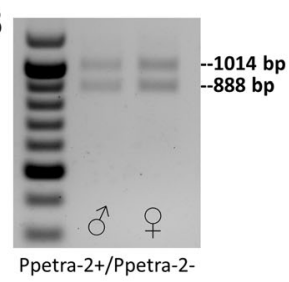

C

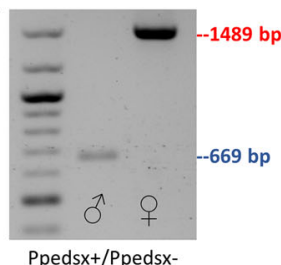

D

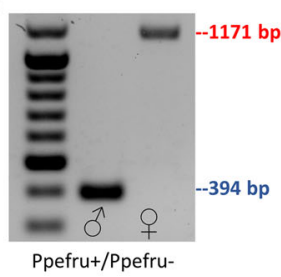

E PpedsxF TRA/TRA-2 binding sites

TCATCAATCAACA
TCTTCAATCAACA
TCTACAATCAACA
TCTACAATCAACA
TCTTCAATCAACA
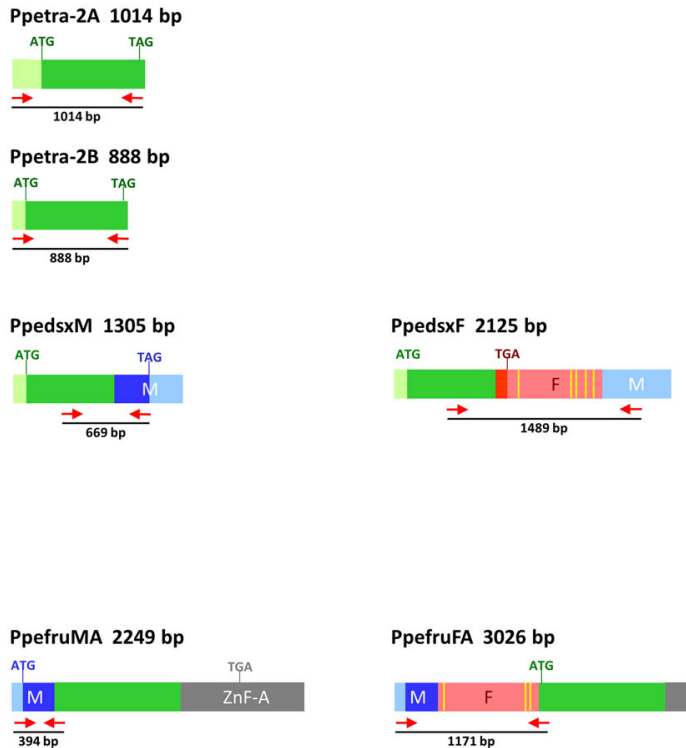

PpefruMc 1915 bp

ATG

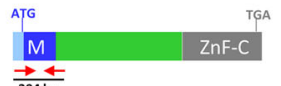

PpefruF TRA/TRA-2 binding sites

TCTGCAATCAACA

TCTTCAATCAACA

TCTTCAATCAACA

Fig. 2 Sex determining genes expression at adult stage in P. perniciosus. The first lane left is 100-bp ladder (NEB). a Positive RT-PCR control with Ppesod+/Ppesod-primer pairs. These PCR primers span a 112-bp long intron of Ppesod gene (genomic amplicon size 450 bp; CDNA amplicon size 338 bp). b Ppetra-2 RT-PCR amplification. c Ppedsx RT-PCR amplification. d Ppefru RT-PCR amplification. Light green boxes represent non-sex specific untranslated regions. Dark green boxes represent non-sex-specific coding regions. Azure boxes represent male-specific untranslated regions. Pink boxes represent female-specific untranslated regions. Blue and red boxes represent male-specific and female-specific coding regions, respectively. Grey boxes represent fru alternative terminal exons encoding for Zinc Finger domains. The position of primers utilized for each gene are indicated by short red arrows. Yellow vertical bars indicate the position of the putative TRA/TRA-2 binding sites. e Putative TRA/TRA-2 binding sites identified in Ppedsx and Ppefru female-specific transcripts 
$2 B$ showed that it encodes for a putative TRA-2 protein with slight amino acid (aa) differences in the $\mathrm{N}$-terminus respect to PpeTRA-2A. A similar tra-2 non-sex-specific splicing pattern was reported in the whitefly Bemisia tabaci, where the two encoded TRA-2 isoforms differ at their N-terminus for a wider region of 123 aa [48].

The RT-PCR analysis of the Ppedsx and Ppefru transcripts revealed that both genes are regulated by sexspecific alternative splicing, as in other insect species (Fig. 2c and d). Notably, in both Ppedsx and Ppefru female-specific transcripts we identified a cluster of putative TRA/TRA-2 binding sites (Fig. 2e). The TRA/ TRA-2 binding site cluster, also known as $d s x$ repeat element (dsxRE), represents one of the first alternative splicing enhancer identified in Drosophila [49] where this regulatory sequence has been shown to be both necessary and sufficient for tra- and tra-2-dependent, female-specific splicing of $d s x$ and fru pre-mRNAs; the activity of TRA and TRA-2 is instead not required for the processing of pre-mRNA of both genes in males, which constitutes the default type of splicing $[49,50]$. In Diptera, the presence of a conserved TRA/TRA-2 binding site cluster in $d s x$ and fru genes is always associated to the presence of the TRA active protein [16].
Encouraged by finding conserved TRA/TRA-2 binding sites in Ppedsx and Ppefru and by the presence of a PpeTRA-2 with a highly conserved RNA binding domain (Additional file 2: Figure S2), we pursued a second strategy to identify the ortholog of tra in P. perniciosus. This approach was based on the hypothesis that also in sand flies the tra gene could regulate its own sex-specific alternative splicing binding a cluster of TRA/TRA-2 binding sites. Therefore, we analyzed the $P$. perniciosus adult transcriptome with the DREG tool of the Emboss Suite (http://emboss.sourceforge.net/) to detect transcripts containing putative TRA/TRA-2 binding sites. We identified an assembled transcript (c23543.g1.i2, 3858 bplong) containing the highest number of TRA/TRA-2 binding sites, with six elements clustered within a 324 bp-long sequence (Fig. 3a) and located between two putative exons encoding for a serine-arginine rich sequence. Using RT-PCR primer pairs spanning the region containing the TRA/TRA-2 binding sites, we were able to amplify two male-specific (M1 and M2) and three female-specific (F1, F2 and F3) cDNA fragments (Fig. 3b), demonstrating that the c23543.g1.i2 transcript undergoes sex-specific alternative splicing regulation, as expected for a tra ortholog. The low abundance female-

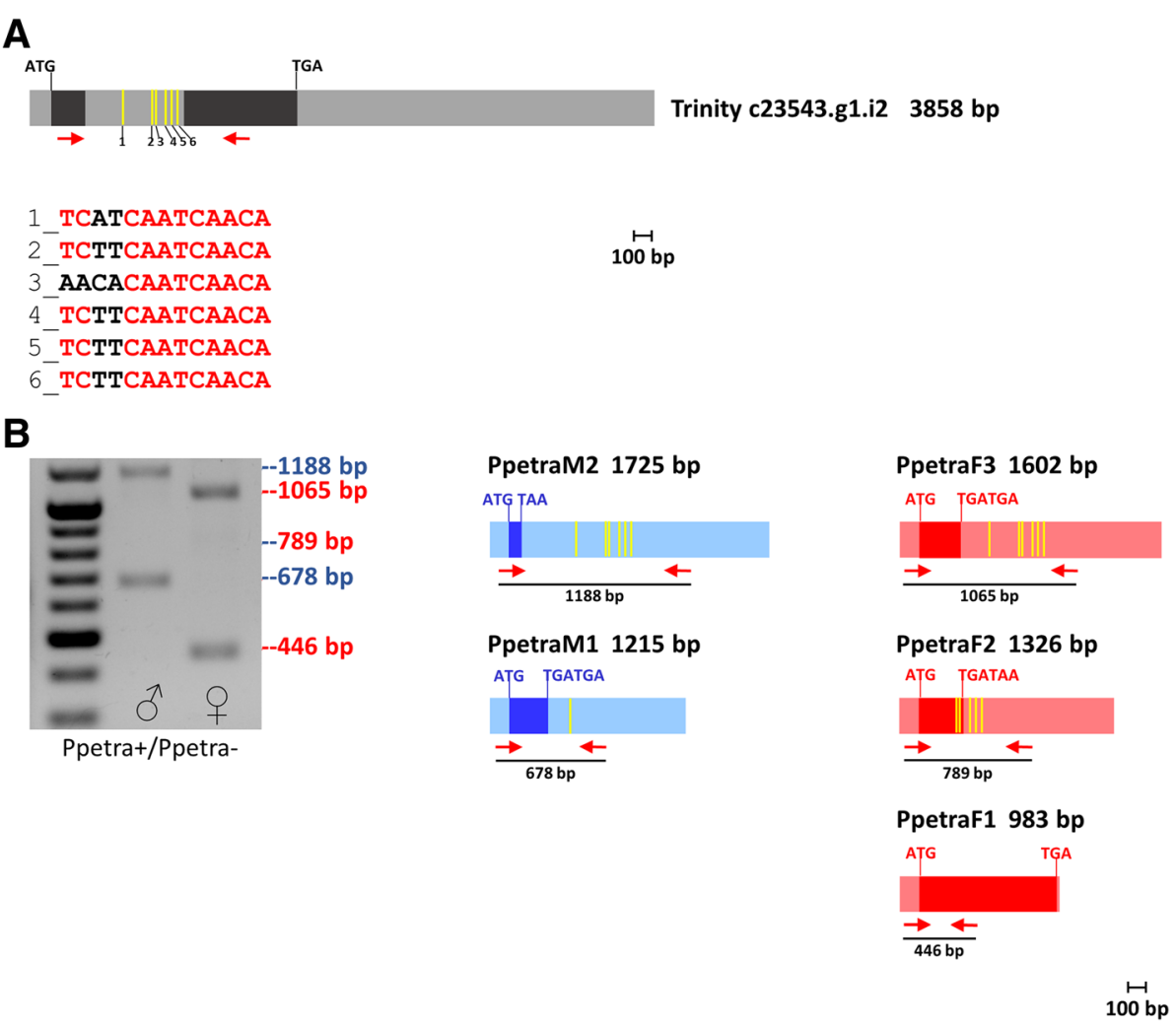

Fig. 3 Ppetra transcripts and expression at adult stage. The first lane left is 100-bp ladder (NEB). a In silico identified Ppetra transcript, containing six putative TRA/TRA-2 binding sites, indicated by yellow vertical bars. b Ppetra RT-PCR amplification. Azure boxes represent male-specific untranslated regions. Pink boxes represent female-specific untranslated regions. Blue and red boxes represent male-specific and femalespecific coding regions, respectively. The positions of primers utilized are indicated by short red arrows 
specific F2 fragment might correspond to partially spliced and/or unstable tra transcripts. Similar splicing pattern of tra was observed in the medfly C. capitata [12].

The five full-length cDNAs were cloned and sequenced after $5^{\prime}$ and $3^{\prime}$ RACE experiments, performed as described in Methods.

The in silico translation of the five cDNAs revealed that M1, M2, F2 and F3 encode for very short polypeptides due to the presence of premature stop codons. Only the female-specific F1 cDNA has a full ORF and encodes for a SR rich sequence (282 aa) containing a short region similar to the TRA Diptera domain (Additional file 2: Figure S9). We named this putative protein as PpeTRA and the corresponding gene as Ppetra. To date, PpeTRA represents the shortest insect TRA protein, excluding the non-autoregulating TRA of D. melanogaster (197 aa) (Additional file 2: Figure S9).

\section{Developmental expression analysis of sex determining genes in $P$. perniciosus}

We performed an RT-PCR analysis on total RNA extracted from samples of mixed sexes from different developmental stages (embryos, larvae of 1st, 2nd and 4th instar and pupae) to analyze the developmental expression pattern of the sex determining genes newly identified in $P$. perniciosus (Fig. 4). We used the Ppesod gene, constitutively expressed in $P$. perniciosus [40], as endogenous positive control, and the same primer pairs of the RT-PCR analyses performed on adult samples, spanning the alternatively spliced regions of tra, tra-2, $d s x$ and fru genes, as reported in Figs. 2 and 3. We found that Ppetra is expressed since embryonic stage, as observed for other dipteran species $[12,17,19]$, producing sex-specific transcripts. We amplified, in all developmental stages, fragments of $446 \mathrm{bp}, 678 \mathrm{bp}$ and $1065 \mathrm{bp}$ corresponding to Ppetra F1, M1 and F3 transcripts, respectively (Fig. 4). In addition, we amplified a low abundance fragment of 1188 bp, corresponding to the Ppetra M2 transcript, expressed at higher level in adult male (Fig. 3b) and a faint fragment of $789 \mathrm{bp}$, corresponding to the Ppetra F2 transcript, whose relative abundance is similar in adult female (Fig. 3b). Ppetra-2 is expressed from the first instar larval stage until adulthood, differently from other dipteran species, such as $C$. capitata and $M$. domestica, where it is

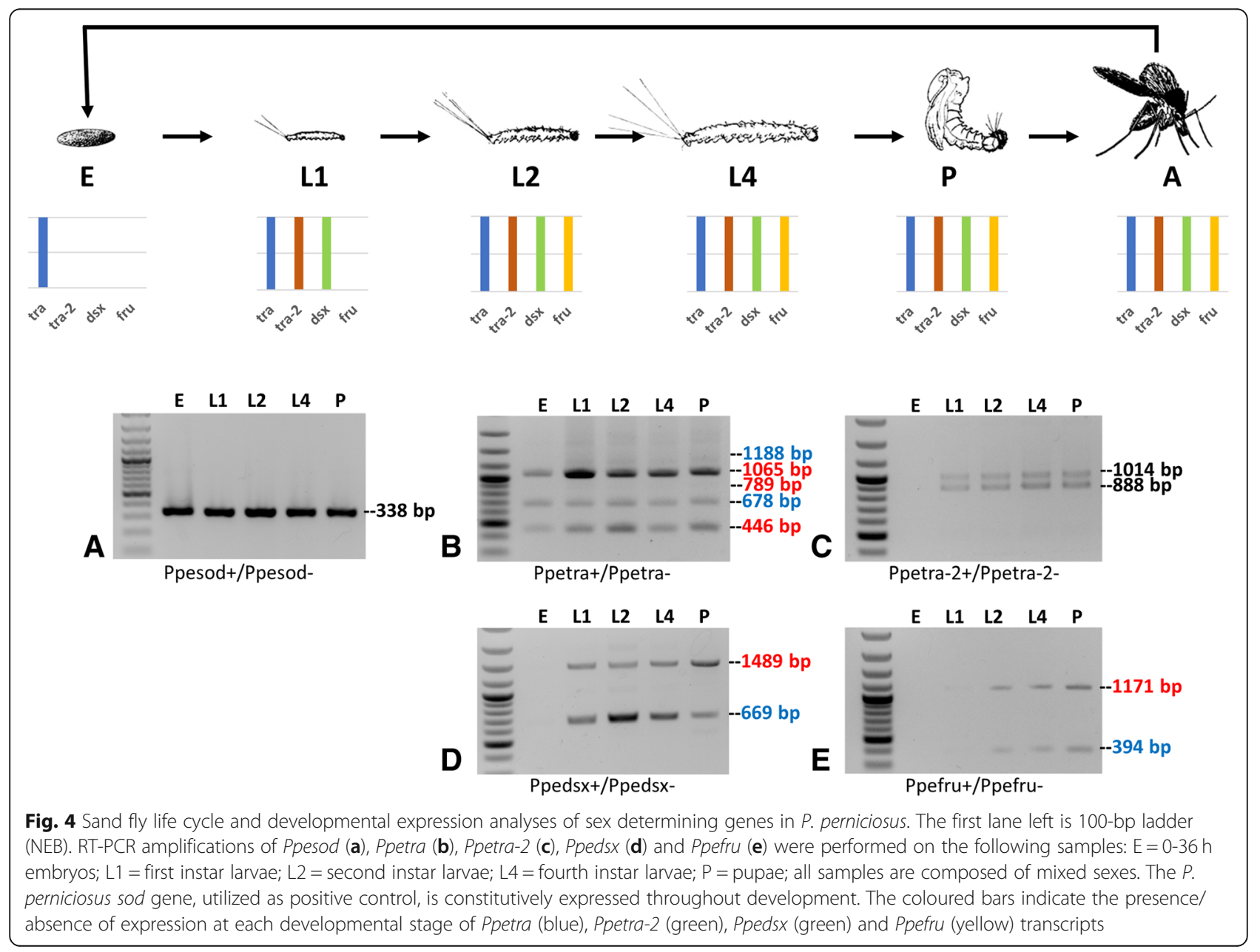


expressed also at embryonic stage $[13,51]$. Both the Ppetra- $2 A$ and $B$ transcripts were detected in all stages but embryos (Fig. 4). Ppedsx and Ppefru are expressed from first-instar larval stage and second-instar larval stages, respectively, until adulthood, both producing sex-specific transcripts by alternative-splicing during development (Fig. 4). Ppedsx developmental expression pattern seems to be different respect to other dipteran species, including Drosophila, C. capitata and the tiger mosquito Aedes aegypti, where $d s x$ is expressed also at the embryonic stage $[24,52,53]$. Conversely, the Ppefru developmental expression pattern is conserved respect to fru-P1 promoter expression pattern observed in Drosophila and in A. aegypti $[23,54]$, with expression starting at late larval stage until adulthood.

\section{Evolution of tra genomic organization and of alternative splicing regulation in Phlebotominae}

The Ppetra gene is the first tra ortholog isolated in a Nematocera species and the shortest tra gene $(1.7 \mathrm{~Kb})$ isolated to date in insects. To study its genomic organization we amplified, cloned and sequenced the 1725 bp fragment corresponding to the Ppetra locus, using a primer pair located in the $5^{\prime}$ and $3^{\prime}$ UTR of Ppetra transcripts and adult genomic DNA. Aligning genomic Ppetra against the five Ppetra cDNA sequences, we reconstructed the exon-intron organization of the Ppetra gene and identified the alternative splicing events producing the Ppetra transcript isoforms (Fig. 5a). The Ppetra gene has four exons and three introns, all with conserved GT-AG boundaries (Additional file 3: Figure S10) . In females, Ppetra produces three transcripts. Exon 1, 2, 3 and 4 are used to produce a mature mRNA corresponding to the F1 transcripts, with an ORF encoding for the 282 aa-long PpeTRA protein. In addition to this, distinct parts of intron 2 are retained in two other transcripts, one by an alternative 3 ' acceptor splicing site (transcript F2) and the other by an intron retention mechanism (transcript F3). In both the F2 and F3 transcripts the presence of in-frame stop codons causes short truncated PpeTRA isoforms. In males, Ppetra produces two transcripts: the M2 transcript is an unspliced transcript because it retains all the introns, while the M1 transcript is produced through an alternative $5^{\prime}$ donor splicing site choice. In the two Ppetra male-specific transcripts, the introduction of premature stop codons leads to short truncated PpeTRA isoforms.

To study the evolution of the genomic organization and alternative splicing regulation in sand flies, we searched the tra orthologs in seven other Phlebotominae species by TBLASTN using PpeTRA as query. For two species, $P$. papatasi and L. longipalpis, genome and transcriptome assemblies were available (PpapI1, PpapI1.4, LlonJ1 and LlonJ1.4; https://www.vectorbase.org/). For the other two Old World sand fly species, $P$. bergeroti and $P$. duboscqi, we assembled a draft genome using sequencing data available at NCBI SRA database and the MINIA genome assembler [55]. In addition, we produced de novo transcriptome assemblies by using all the available sequencing data (up to March 2018 - NCBI SRA database) for L. longipalpis and for two New World species, L. (Nyssomyia) umbratilis and L. (Nyssomyia) neivai using the Trimmomatic tool and the Trinity de novo assembler [56-58] (see Additional file 15: Supplementary Methods).

By querying PpeTRA against the genomes and transcriptomes of the Phlebotominae species, we identified the tra ortholog in P. papatasi (Ppatra), P. bergeroti (Pbetra) and P. duboscqi (Pdutra); however, this approach could not identify any tra ortholog in the genomes/transcriptomes of the three New World sand fly species (L. longipalpis, L. (Nyssomyia) umbratilis and $L$. (Nyssomyia) neivai). Furthermore, neither the TRA/ TRA-2 binding sites in silico approach, that led to the identification of Ppetra, nor a molecular approach in $L$. longipalpis by touch down RT-PCR with degenerated primers designed on the alignment of Ppetra and Ppatra sequences (data not shown) could identify tra in New World sand flies. Similarly, Geuverink and Beukeboom [39] identified in silico a putative tra gene in the Old World sand fly species $P$. papatasi and reported its apparent absence in the New World sand fly $L$. longipalpis [39]. We performed a BLAST analysis using the Ppetra gene coding sequence as query to search eventual tra orthologs in other Nematocera species currently available in public databases (NCBI, VectorBASE), obtaining no result.

During the evolutionary history of the insect tra gene, multiple gene loss events have been hypothesized (within Diptera, Lepidoptera, Coleoptera and Strepsiptera) $[23,39,59]$. Our results confirm this hypothesis and support the existence of a tra gene loss event within Phlebotominae, after the split in Phlebotomus (New World) and Lutzomyia (Old World) sand flies. The common ancestor of these two lineages is thought to have occurred about 250 million of years ago (MYA) (Triassic period) and their separation is dated back to the breakup of Pangaea, about 200 MYA [60].

We reconstructed gene models for the $P$. papatasi, $P$. bergeroti and $P$. duboscqi tra orthologs (Additional file 3: Figures S11-S13), which encode for a 311 aa-long SRprotein with $61 \%$ identity respect to the PpeTRA and missing, as in P. perniciosus, a conserved TRACAM domain (Additional file 4: Figure S14). The four Phlebotomus tra genes revealed a conserved genomic organization with four exons and three introns, with small differences in exons/introns lengths (Fig. 5b). In the intron 2 of $P$. papatasi, $P$. bergeroti and $P$. duboscqi, we identified, as in $P$. 

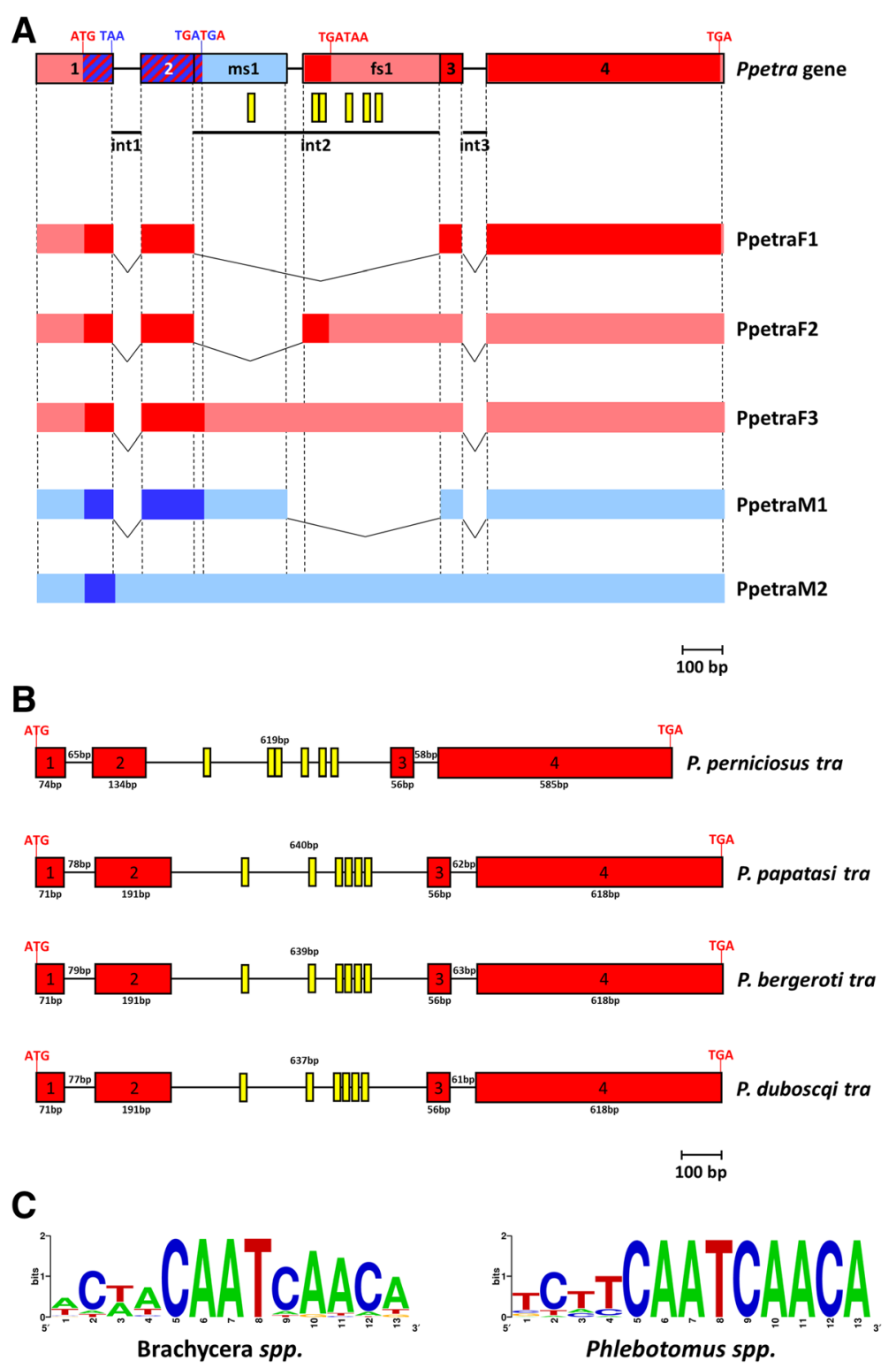

Fig. 5 P. perniciosus tra genomic organization and evolution. a Ppetra gene locus and sex-specific transcripts. Azure boxes represent male-specific untranslated regions. Pink boxes represent female-specific untranslated regions. Blue and red boxes represent male-specific and female-specific coding regions, respectively. Striped blue-red boxes represent coding regions utilized both in the male and female sex. Yellow vertical bars indicate the position of the putative TRA/TRA-2 binding sites. b Comparison of tra gene structures in Phlebotomus species. c WebLogo consensus sequence of the putative TRA/TRA-2 binding sites identified in Phlebotomus tra species and of TRA/TRA-2 binding sites of Brachycera tra genes

perniciosus, six conserved TRA/TRA-2 binding sites (Fig. 5c). To study the alternative splicing regulation of the tra gene in Phlebotomus species, we compared the intron sequences of the four species (Additional file 5: Figure S15). As in P. perniciosus, all tra introns exhibit conserved GTAG terminal dinucleotides. Intron 2, which is subjected to sex-specific alternative splicing in $P$. perniciosus, has a putative conserved alternative splicing sites (SS) also in $P$. papatasi, P. bergeroti and P. duboscqi. In the four species, the $5^{\prime}$ donor SS of intron 2 seems to be weak and suboptimal, while the 3' acceptor SS is a canonical strong splicing site. Finally, all the four species have a strong canonical male-specific alternative $5^{\prime}$ donor SS at about $230 \mathrm{bp}$ downstream the exon 2 (Additional file 5: Figure S15). These findings led us to suppose that in $P$. perniciosus, as well as in the other three Phlebotomus species, the malespecific splicing of the tra pre-mRNA represents the default splicing mode. In contrast, in females, the repression of the male-specific $5^{\prime}$ donor SS of intron 2 is most probably due to the binding of TRA and TRA-2 proteins on the TRA/TRA-2 binding site cluster, leading to the usage of the upstream $5^{\prime}$ donor SS to form to the female- 
specific tra transcript, thus producing a functional TRA only in females. This hypothesis on the conserved splicing regulation was confirmed in $P$. papatasi by RT-PCR on adult RNA from males and females (Additional file 6: Figure S16).

Figure 6 shows a comparison of the tra genomic locus among insect species. Despite differences in exon number and intron length, the sex-specific splicing regulation of the tra gene exhibits a striking conservation. In all the considered species, including $P$. perniciosus, an alternative $5^{\prime}$ donor SS choice leads to a full TRA protein only in the female sex. To study the protein organization, we compared PpeTRA with other arthropod TRA proteins (Fig. 7a). PpeTRA exhibits similar domain organization respect to insect TRAs, with a DIPTERA domain located within the RS domain, as observed also in TRA of
Lucilia cuprina (LcTRA), Cochliomyia hominivorax (ChTRA) and Glossina morsitans (GmTRA). At the same time, PpeTRA misses the TRACAM domain; the Ppetra regions corresponding to the last 31 nucleotides of exon 2 and to the first 45 nucleotides of exon 3 (upstream and downstream the Ppetra sex-specifically regulated intron, respectively) encode for a PpeTRA protein portion that exhibits only 8 out of 25 conserved amino acids respect to the insect TRACAM domain (Fig. 7b).

In conclusion, despite the absence in sand fly TRAs of the TRACAM domain, supposedly involved in the autoregulation of the tra gene $[4,14]$, the conserved sand fly tra structure, the conserved sex-specific alternative splicing regulation and the presence of a highly conserved TRA/TRA-2 binding site cluster in the sex-specifically regulated tra intron strongly support the hypothesis of

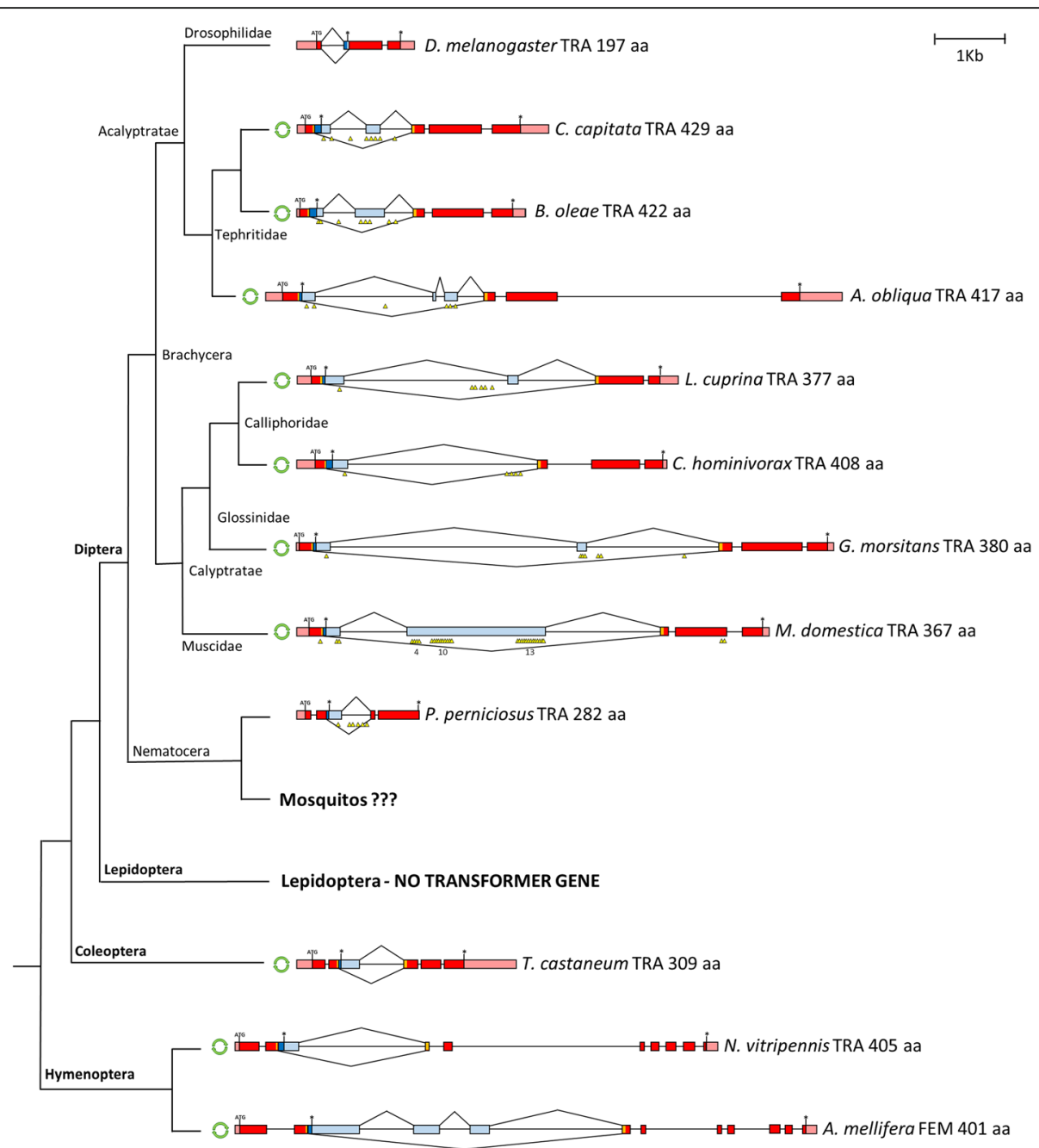

Fig. 6 Phylogenetic relationship, genomic structure and sex specific splicing regulation of transformer orthologues in insects. Pink boxes indicate untranslated regions; azure boxes indicate untranslated male-specific regions; red boxes indicate female-specific exon/regions; blue boxes indicate male-specific coding regions; yellow boxes indicate the position of the tra region encoding for the TRACAM domain. Translational start (ATG) and stop $\left(^{*}\right)$ sites are indicated. Yellow triangles indicate the position of the putative TRA/TRA-2 binding sites (for $M$. domestica is reported also the number of TRA/TRA-2 bindings sites). Green double circular arrows indicate autoregulation of the tra ortholog 


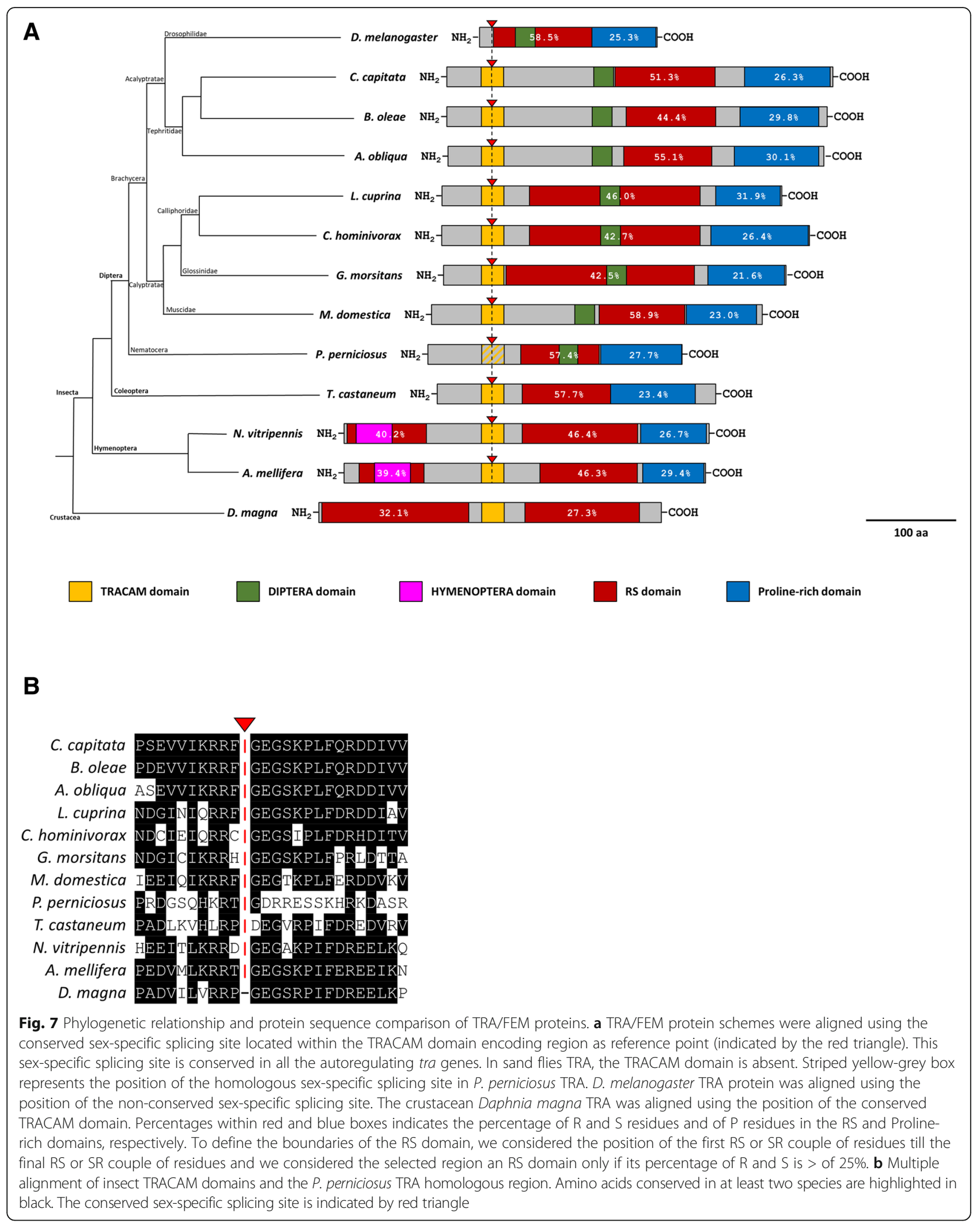


the autoregulation of the tra gene in Phlebotomine sand flies, as observed for other dipteran and non-dipteran species.

Considering the absence of the TRACAM domain in sand flies, we hypothesized two possible mechanisms to explain tra autoregulation in insects: 1) the autoregulation of tra is controlled by a different TRA region still to be identified; 2) a second auxiliary factor could be involved in the autoregulation of the tra gene, together with TRA and TRA-2 proteins. Future in vivo functional analyses might help to unravel this key point and to get insight on the still unclear mechanism of the tra gene autoregulation in insects.

\section{Evolution of tra-2 genomic organization in Phlebotominae}

tra-2 is a single-copy gene that has been characterized in D. melanogaster $[61,62]$ and in several other dipteran species such as $D$. virilis [63], the house fly $M$. domestica [51], the tephritids C. capitata [13, 64] and 12 Anastrepha species [65], the calliphorid Lucilia cuprina [20], and the Nematocera sciarid species Sciara ocellaris and Bradysia coprophila [66]. In these species, tra-2 is transcribed during development in both sexes, producing an RNA-binding protein with two RS domains flanking an RRM domain. TRA-2 RRM is followed by a 19 aa-long linker region, which is a distinctive and unique feature of the TRA-2 proteins [67]. Within Brachycera suborder, TRA-2 is required for the sex-specific splicing regulation of the $d s x$ and fru genes and, outside Drosophilidae, it is also involved in the autoregulation of female-specific alternative splicing of the tra gene [13, 19, 51, 65, 68].

Using the available genomic resources of $P$. papatasi, the assembled draft genomes of $P$. bergeroti and $P$. duboscqi and the identified putative TRA-2 proteins of $P$. perniciosus, we reconstructed the partial putative exon-intron structure of tra-2 of the Old World sand flies consisting of 4 exons and 3 introns (Additional file 7 : Figures S17-19). In addition, we identified in P. papatasi, $P$. bergeroti and $P$. duboscqi a putative alternative $5^{\prime}$ donor splicing site located downstream of the $5^{\prime}$ donor splicing site of the exon 1 of tra-2, which is conserved in $P$. perniciosus where it leads to the production of the PpeTRA-2B isoform. As these species belong to different subgenera (Phlebotomus and Larroussius), this suggests that a similar non-sex-specific alternative splicing event could be conserved also in other Old World sand flies (Additional file 7: Figures S17-19). More in general, among dipteran, tra-2 shows an overall conservation of exons encoding for functional domains and both RRM and RS1 domains are encoded by several exons. In Nematocera Old World sand flies, the RS1 domain is encoded by a unique exon, while the RRM domain and the linker region are organized in two exons (Fig. 8).
As observed for the tra ortholog, tra-2 seems to be absent in transcriptome and genome assembly of the New World sand fly L. longipalpis. However, differently from tra, we found well conserved TRA-2 encoding transcripts, missing the $\mathrm{N}$-terminus coding regions, in L. umbratilis and $L$. neivai. This finding suggests that the tra-2 ortholog could be present also in L. longipalpis species but not correctly assembled in the $L$. longipalpis released transcriptome/genome assemblies as well as in the L. longipalpis transcriptome assembled in the present study. In (Additional file 8: Figure S20) the multiple alignment of sand fly putative TRA-2 protein is reported. A very well conserved RRM + linker region and RS1 region are present in all the species analyzed. A RS2 region was detected only in P. perniciosus, $L$. umbratilis and L. neivai. The high percentage of conserved residues of the RS2 region (22/51) suggests its conservation also in other Phlebotomus species (Additional file 8: Figure S20).

In summary, with our work we identified for the first time the tra-2 gene in sand flies. Previously, the tra-2 ortholog of Nematocera was characterized only in the sciarid species $S$. ocellaris and B. coprophila and in the mosquito An. gambiae and Ae. aegypti, where two and four orthologs were found, respectively. In S. ocellaris and B. coprophila TRA-2 is highly conserved and shows conserved sex-determination function when expressed in Drosophila [66]. Conversely, putative TRA-2 identified in mosquitoes seem to be divergent respect to other dipteran TRA-2 and possibly not involved in the control of sex-specific splicing of $d s x$ and fru targets [23, 24]. Recent functional tests by transgene-mediated RNAi against Ae. aegypti tra-2 orthologs have shown no female-to-male sex reversion, as obtained in tra-2 RNAi functional studies in Brachycera species; instead, a novel femalespecific zygotic lethality was observed. This finding supports the hypothesis that tra-2 does not play a conserved role in Ae. aegypti sex determination, while it controls a novel female-specific vital functions which need to be clarified [69]. Here we show that, as for tra-2 of sciarid species, in sand flies tra-2 encodes for a protein conserved in its structure and domains, suggesting a conserved role in the sex determination through sex-specific alternative splicing regulation of both $d s x$ and fru downstream target genes. In addition, we propose that tra- 2 could be involved in the autoregulation of the tra gene also in Old World sand flies. The absence of a tra ortholog in New World sand flies poses a very interesting problem about the function of tra-2 in these species and about the evolution of the alternative splicing regulation of $d s x$ and fru genes. 


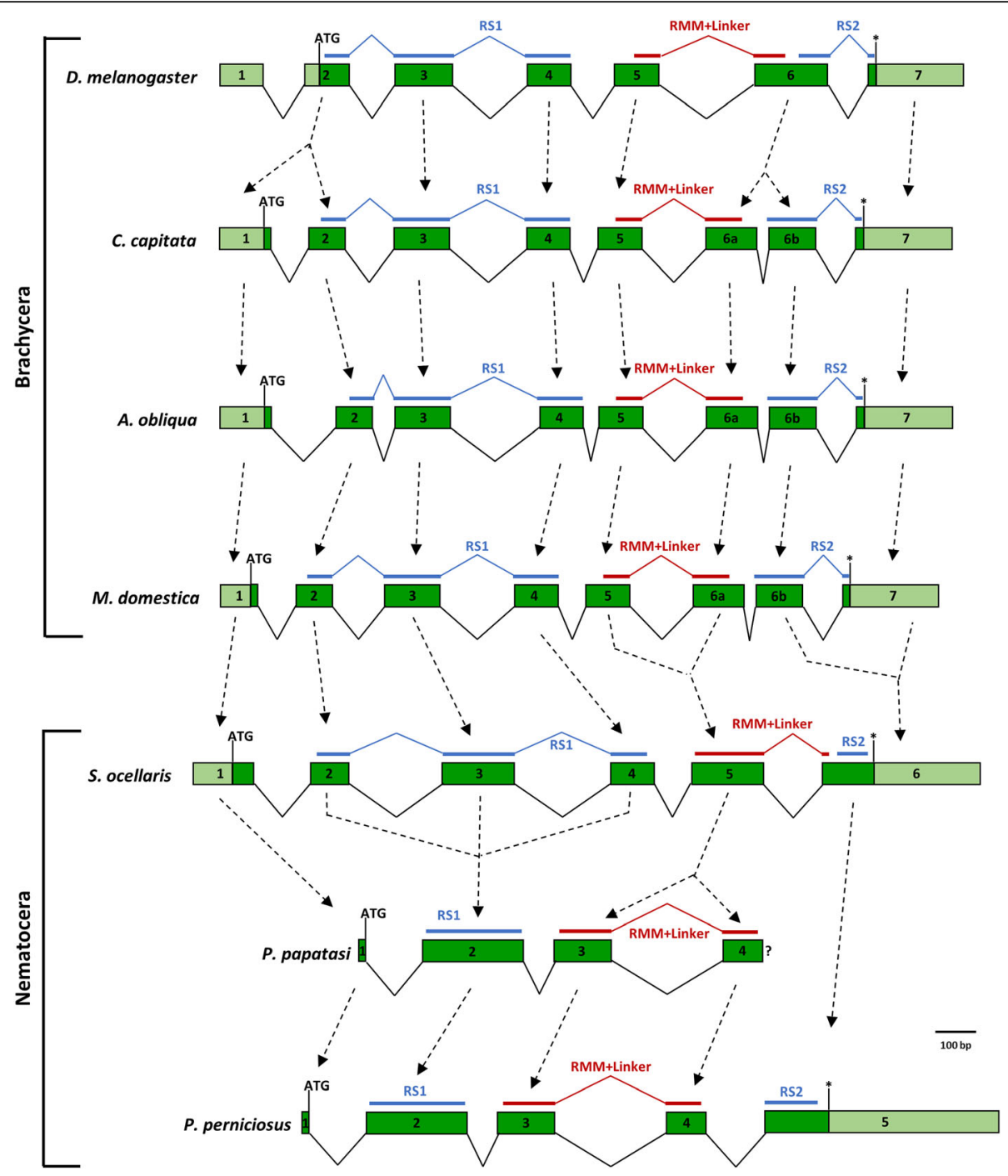

Fig. 8 Comparison of genomic structures of dipteran tra-2 genes. Green boxes represent coding regions; light green boxes represent untranslated regions; asterisks indicate the position of stop codons. The exon portions encoding for RS domains, RRM domain and linker region are indicated. Introns are not to scale. $P$ perniciosus tra-2 intron-exon organization has been predicted by comparison with P. papatasi partial tra-2 gene

\section{Evolution of $d s x$ and fru genomic organization and} alternative splicing regulation in Phlebotominae

To study the evolution of the genomic organization and of the alternative splicing regulation of $d s x$ and fru genes in the sand flies, we aligned DSXs and FRUs of P. perniciosus, $P$. papatasi and $L$. longipalpis against the genome sequences of $P$. papatasi and L. longipalpis using TBLASTN (Additional file 9: Figures S21-S24, Additional file 15: Supplementary Methods). By manually refining the exonintron junctions, we obtained the structure of the genes. Compared with the orthologs in D. melanogaster, An. gambiae and Ae. aegypti, we observed an overall conservation of the exon/intron organization and of the alternative splicing regulation in sand flies (Additional file 9: Figures S25-S26).
In particular, as observed in other dipteran species [24, $26,52,53,70,71]$, in sand flies $d s x$ is organized in 4 exons spread over a large genomic region $(146 \mathrm{~Kb}$ in $P$. papatasi and at least $191 \mathrm{~Kb}$ in L. longipalpis). Exon one, which contains the ATG signal, encodes for the DSX OD1 domain and is followed by the second exon encoding for the nonsex-specific part of the DSX OD2 domain. Exon three is female-specific and encodes for the female-specific DSX Cterminus. Exon four is present in transcripts of both sexes as 3'untranslated region in females and encoding for malespecific DSX C-terminus in males (Additional file 9: Figure S25). Interestingly, the nucleotide sequence of the region surrounding the 3 ' acceptor female-specific splicing site of the $d s x$ gene is strictly conserved among Phlebotomus species (Additional file 10: Figure S27). A similar observation 
was recently reported by Kyrou and colleagues [72] for Anopheles mosquito. This region was utilized to develop a gene drive-based population suppression strategy resulted very effective in small scale caged experiments [72]. This finding suggests that also the $d s x$ gene of Phlebotomus species could be an ideal target to develop future similar strategies for sand fly control in field.

The fru gene in sand flies is organized in eight exons distributed over a very large genomic region (at least 125 $\mathrm{Kb}$ in $P$. papatasi and $213 \mathrm{~Kb}$ in L. longipalpis). Exons one and two (named S1 and S2, respectively) are common and female-specific respectively, with exon S1 encoding the male-specific $\mathrm{N}$-terminus of FRU and exon S2 utilized only in females as $5^{\prime}$ untranslated region. Exons three and four (named $\mathrm{C} 1$ and $\mathrm{C} 2$ ) encode for the BTB domain, while exons five to seven (named C3, C4 and C5) encode the poorly conserved Connector region. The terminal exon eight encodes for a zinc-finger domain of type C (Additional file 9: Figure S26). Using the P. perniciosus fru $\mathrm{ZnF}-\mathrm{A}$ and the $D$. melanogaster protein sequence of ZnF-B as queries, TBLASTN analysis of the genomic scaffold 549 of $P$. papatasi PpapI1 assembly, containing the fru exon eight, revealed the presence of putative exons encoding very well conserved $\mathrm{ZnF}$ domains. This finding suggests that also in sand flies the fru gene could encodes for multiple FRU isoforms by alternative splicing at the 3'end of the primary transcripts (data not shown).

Figure 9a shows a schematic representation of the sexspecifically regulated regions of both $d s x$ and fru genes in D. melanogaster, An. gambiae, Ae. aegypti, P. papatasi and $L$. longipalpis. As for most of the Brachycera species, in Drosophila $d s x$ and fru sex-specific alternative splicing is achieved through two different mechanisms. For $d s x$, a $3^{\prime}$ alternative acceptor splicing site choice coupled with alternative polyadenylation leads to sexspecific transcripts with different $3^{\prime}$ ends encoding for sex-specific DSX C-termini (Fig. 9a) [73]. For fru, a 5' alternative donor splicing site choice leads to sexspecific transcripts with different $5^{\prime}$ ends. In males, a male-specific FRU, with a unique $\mathrm{N}$-terminus is obtained through the usage of an ATG signal present in the fru male-specific exon (Fig. 9a) [50]. In females, a stop codon in the female-specific exon produces a transcript with a very short open reading frame, probably not translated (Fig. 9a). For both the genes, the male-specific splicing represents the default mode. In female, the presence of TRA and the consequent formation of the TRA/ TRA-2 complex which binds the TRA/TRA-2 binding sites in $d s x$ and fru female-specific exons, promotes female specific splicing $[50,73,74]$.

In Nematocera, $d s x$ and fru orthologs have been characterized in few species including the mosquito $A n$. gambiae and Ae aegypti [23-26, 54, 75, 76]. While sexspecific splicing regulation of the fru orthologs in both mosquito species is very well conserved respect to Drosophila (Fig. 9a) [23, 25], for $d s x$ a different mechanism was described in each species. In An. gambiae, malespecific DSX is obtained by skipping the female-specific $d s x$ exon; instead the male-specific exon sequence is used in females as $3^{\prime}$ untranslated region due to the absence of an alternative polyadenylation signal [26]. In Ae. aegypti, $d s x$ presents two female-specific exons, like in Sciaridae [75], that are escaped in males. In females, inclusion of both or only the second female-specific exon results in two isoforms. In both Ae. aegypti and An. gambiae, due to the absence of an alternative polyadenylation signal in the female-specific $d s x$ exons, male-specific exons are used as $3^{\prime}$ untranslated region [24].

In sand flies, fru has a very well conserved alternative splicing regulation, identical to $D$. melanogaster and mosquitoes, based on a 3' alternative acceptor splicing site choice mechanism. The $d s x$ gene alternative splicing regulation is instead similar to An. gambiae regulation, with an exon-skipping of a female-specific cassette exon only in males and with the males-specific exonic sequence, present also in female-specific transcripts, used as untranslated region (Fig. 9a).

The analysis of $d s x$ and $f r u$ female-specific exons in $P$. perniciosus, $P$. papatasi and $L$. longipalpis revealed the presence of clusters of the cis-acting regulatory element named TRA/TRA-2 binding sites (Fig. 9b). In particular, we identified nine elements in $P$. perniciosus (six located in the PpedsxF and three in the PpefruF transcripts), six elements in $P$. papatasi (five located in the PpadsxF and one in the PpafruF transcripts) and 11 elements in $L$. longipalpis (five located in the Llods $x F$ and six in the LloefruF transcripts) (Additional file 11: Table S2). The identified TRA/TRA-2 binding sites are organized in clusters of at least three elements, except for the single element identified in the PpafruF female-specific exon (Additional file 9: Figure S23A).

As for P. perniciosus (subgenus Larroussius), in both $P$. bergeroti and $P$. duboscq $i$ the fru $\mathrm{S} 1$ exon, encoding for the putative male-specific FRUM N-terminus, is followed by a putative female-specific S2 exon containing three conserved TRA/TRA-2 binding sites (Additional file 12: Figures S28-S31). Similarly, the $d s x$ female-specific exon in $P$. bergeroti and P. duboscqi shows six clustered TRA/TRA-2 binding sites, as observed in the other sand fly species (Additional file 12: Figures S30-S31). The absence of a cluster of TRA/TRA-2 binding sites in P. papatasi fru could be also due to an incorrect assembly of the corresponding fru genomic region.

Intra-species alignment of the TRA/TRA-2 binding sites in sand flies revealed high sequence conservation. In Fig. 9b, the WebLogo (http://weblogo.berkeley.edu/) consensus sequences for TRA/TRA-2 binding sites of various dipteran species are reported. Differently from other 

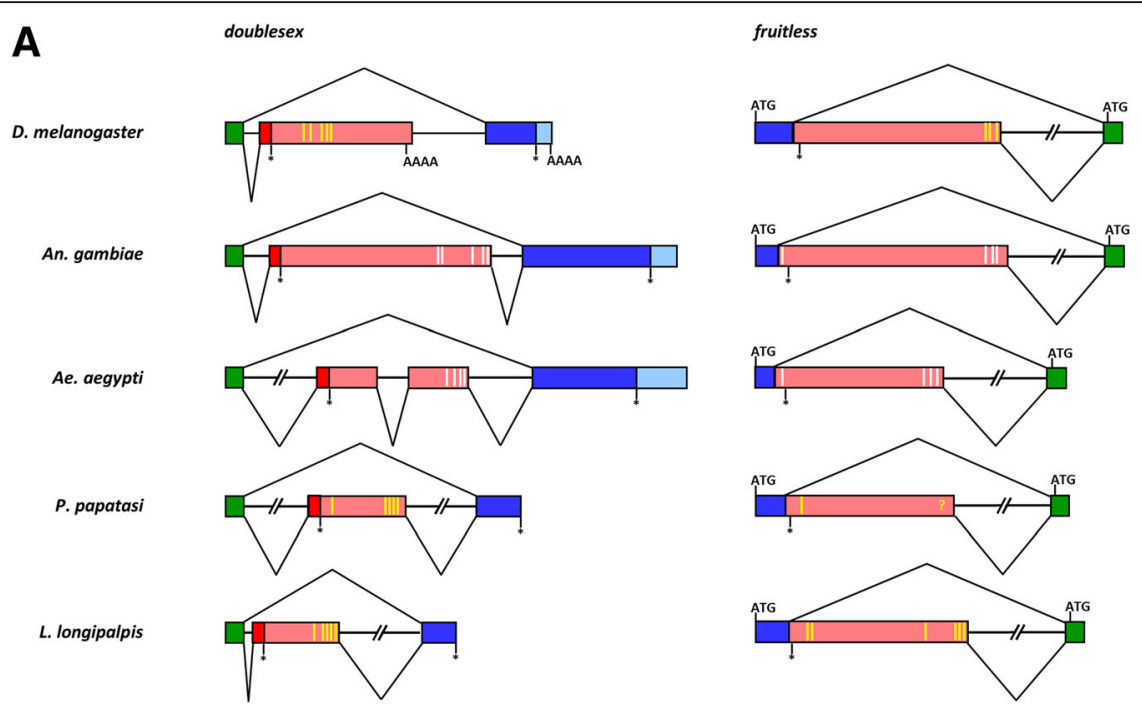

B

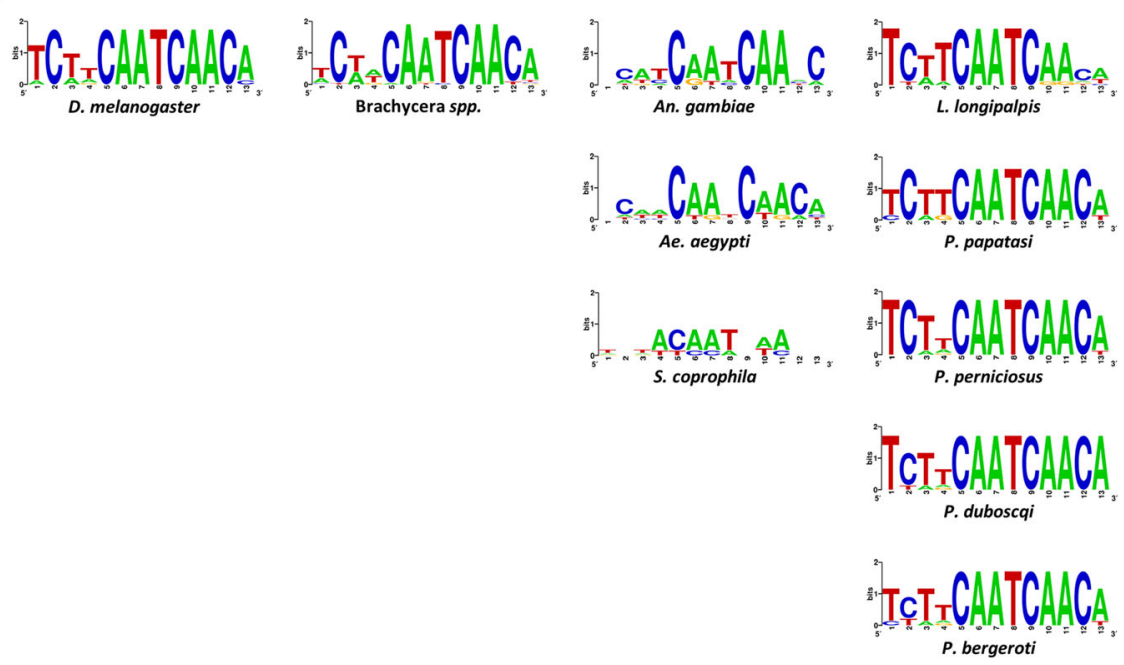

Fig. 9 Evolution of sex-specific alternative splicing regulation of $d s x$ and fru gene. a Comparative schematic representation of sex-specifically regulated regions of $d s x$ and fru genes in $D$. melanogaster, mosquito and sand fly species. Green boxes represent non-sex-specific coding regions; azure boxes represent male-specific untranslated regions; pink boxes represent female-specific untranslated regions; blue and red boxes represent male-specific and female-specific coding regions, respectively; yellow vertical bars indicate the position of the putative TRA/TRA-2 binding sites; white vertical bars indicate the degenerated mosquito putative TRA/TRA-2 binding sites. $\mathbf{b}$ WebLogo consensus sequence of the putative TRA/ TRA-2 binding sites of Brachycera and Nematocera species. Within sand flies, L. longipalpis exhibits the less conserved TRA/TRA-2 binding sites, as expected for a species with upstream regulator/s of the alternative splicing of $d s x$ and fru genes different from tra

Nematocera species, such as the mosquitoes An. gambiae and Ae. aegypti and the sciarid fly S. ocellaris, within each 13-bp long TRA/TRA-2 binding sites of sand flies we observed an invariable "core" of $8 \mathrm{bp}$ (CAATCAAC) and a low variability, as observed in Drosophila, in the first four bases and in the terminal base of the element. In a previous work, we proposed that in mosquitoes, the degeneration of the putative TRA/TRA-2 binding sites is related with the absence of the tra ortholog and with the low level of TRA-2 conservation, suggesting that different upstream regulators are involved in the control of $d s x$ and fru genes in this Nematocera species [23]. Conversely, the high conservation of the TRA/TRA-2 binding sites in Phlebotomus, which resembles the sequence conservation level of the TRA/TRA-2 binding sites observed in $d s x$ and fru genes of Brachycera, indicates that these elements, located in untranslated regions of both genes, are under strong selective pressure. Overall, our findings suggest that also in sand flies TRA and TRA-2 


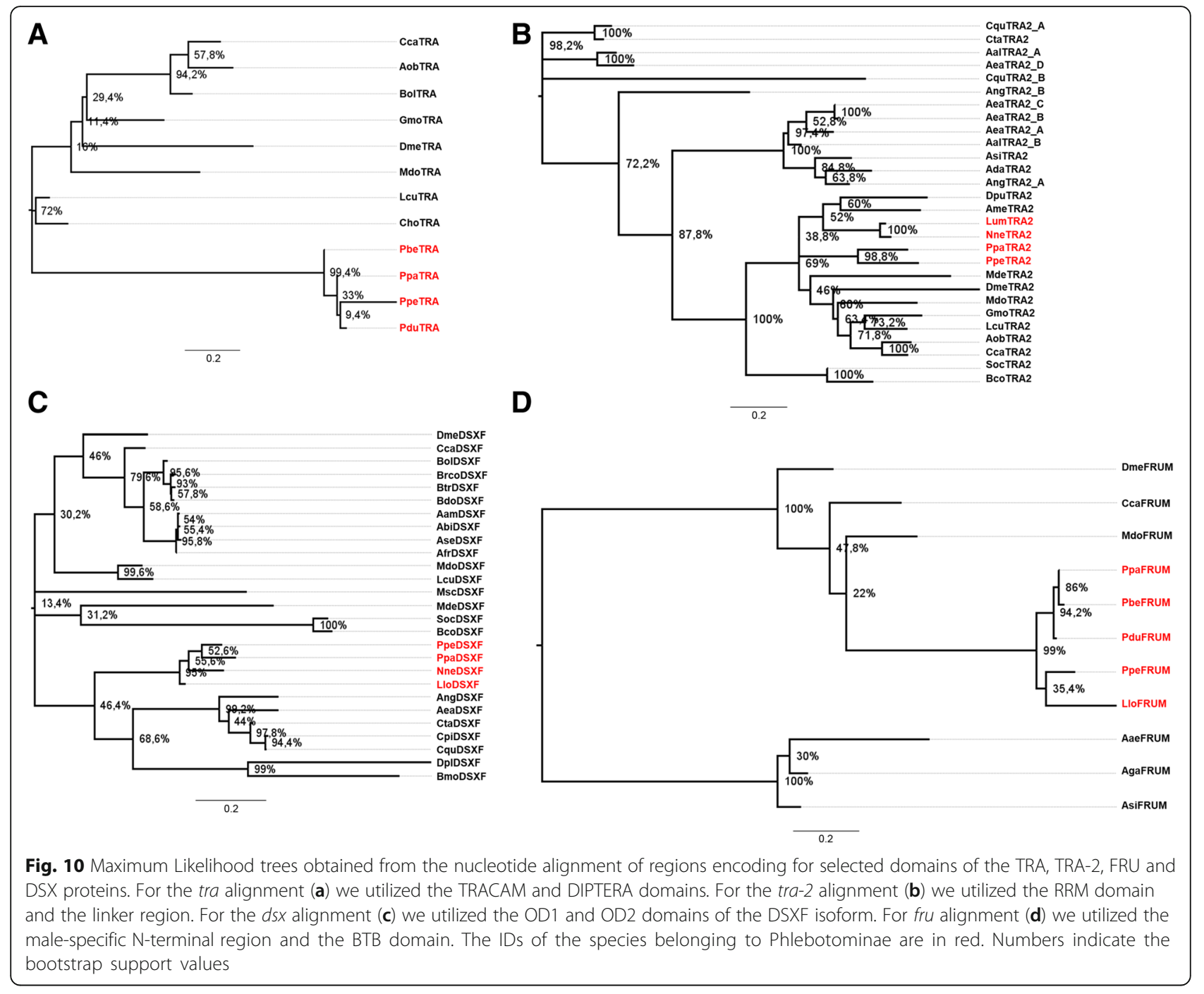

are involved in the regulation of the sex-specific alternative splicing of $d s x$ and fru genes, as observed in Brachycera.

\section{Phylogeny and selection at sex determination genes in sand flies}

Figure 10 shows the Maximum Likelihood trees obtained from nucleotide alignments of the regions encoding for selected domains of the TRA (Fig. 10a), TRA2 (Fig. 10b), DSX (Fig. 10c) and FRU (Fig. 10d) proteins of P. perniciosus and other species (see Methods and Additional file 13: Table S3). For tra and $d s x$, phylogenies segregate sequences in general agreement with the species phylogeny, whereas for tra-2 and fru the sequences of sand flies and mosquitoes are not placed in the same group.

We investigated natural selection at molecular level as the ratio between the mean nonsynonymous and synonymous substitution rates $(\omega)$ of the examined coding regions. To check if the $\omega$ ratios differed significantly among the tree branches, we compared one-, two- and three-ratio models [77] for each gene. The one-ratio model assumes an equal $\omega$ for all the branches, whereas the two- and three-ratio models consider two and three different $\omega$ values, respectively. In addition, we tested the branch-site model that assumes positive selection at specific sites within specific the tree branches $[78,79]$. The results obtained, and the statistical significance of each comparison, are shown in (Additional file 14: Table S4). Overall $\omega$ is always lower than 1 , showing that purifying selection acts on these genes (Additional file 14: Table S4).

The evolutionary analysis of the TRACAM and DIPTERA tra domains shows that the one-ratio model best fits the data $(\omega=0.0864)$ and the absence of positive selection.

Within the tra-2 RMM and linker domains, the tworatio model is better supported than the one- and threeratio models, with the mosquito branch showing the 
lowest $\omega$ value $(0.0407)$ when compared to the other branches $(\omega=0.0738)$. The branch-site model identifies two positively selected sites within the branch that does not include mosquitoes; however, the comparison with its null model is not statistically supported.

Within the $d s x$ OD1 and OD2 domains, the one-ratio model can be excluded in favor of the two- and threeratio models. The two-ratio model fits the data better than the three-ratio model, showing more relaxed selective constraints of the Phlebotominae branch $(\omega=0.0732)$ when compared to the other branches of the tree $(\omega=$ 0.0367). The branch-site model that assumes positive selection at specific sites within the Phlebotominae branch identifies three sites with $\omega$ significantly higher than 1 (Additional file 14: Table S4); however, the comparison

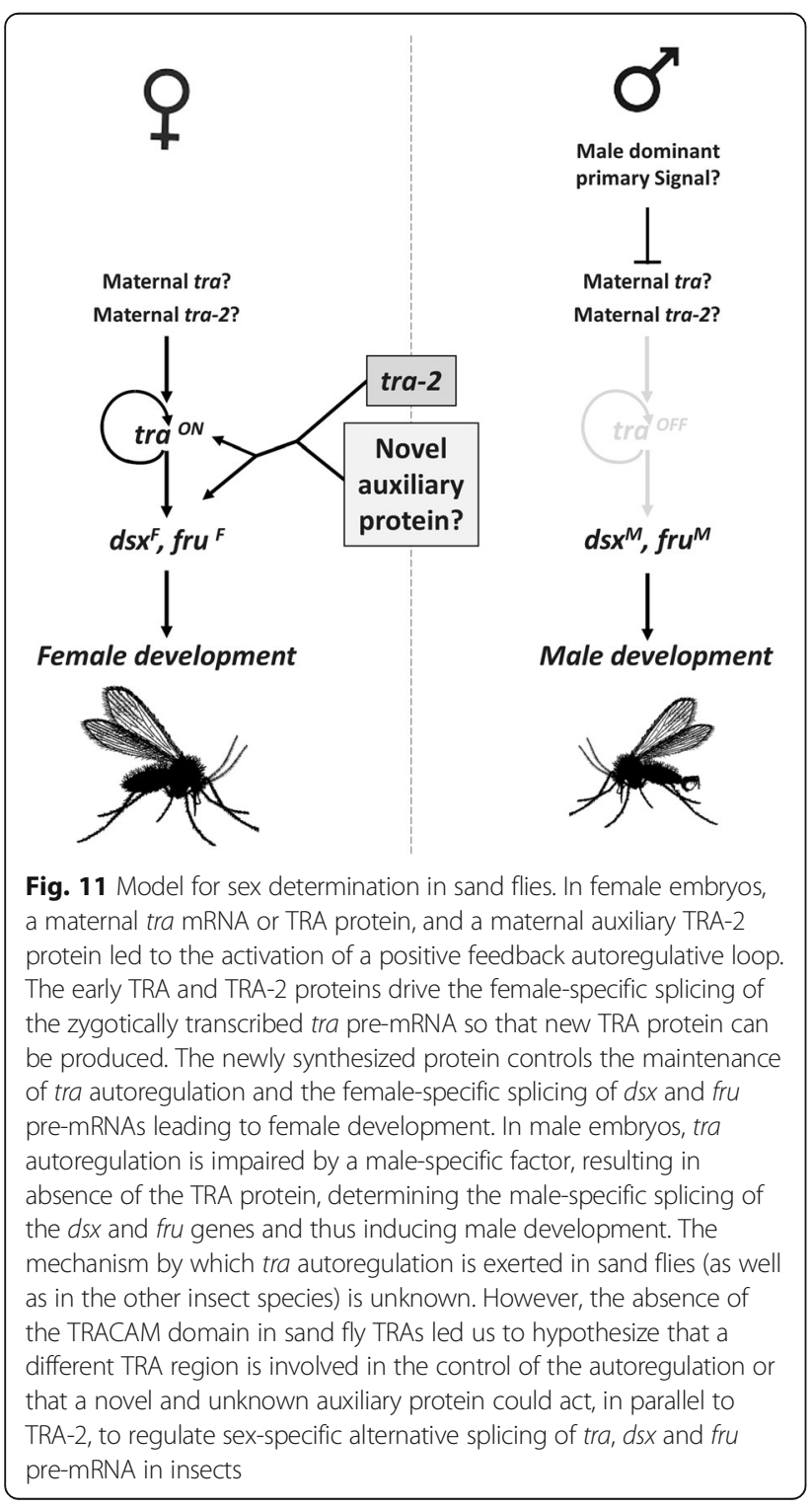

with the null model that assumes absence of positive selection is not statistically significant.

Finally, within the fru male-specific domain, the threeratio model is supported better than the one- and tworatio models, showing a relaxation of the selective constraints within the Phlebotominae branch $(\omega=0.192)$ when compared to the mosquito branch $(\omega=0.1033)$ and to the branch including Drosophila, Ceratitis and Musca $(\omega=0.0288)$. Site and branch-site models do not show evidence of positive selection.

In conclusion, the analysis of the evolutionary pressure acting on the examined sex-determination genes shows evidence of strong purifying selection. However, different selective constraints act on specific branches of the $d s x$ and fru and tra-2 genes, whereas the evolutionary rates of the tra genes appear more uniform.

\section{Conclusions}

Our results permit to hypothesize a model for the sex determination cascade of Phlebotominae sand flies as shown in Fig. 11, which represents the first complete and conserved sex determination cascade observed in a Nematocera species. In particular, we identified all the key sex determining genes, that in Fig. 1 are represented by question marks and, for the first time in a Nematocera species, we identified the homolog of the transformer gene. In addition, our data strongly suggest the conservation of the autoregulation of the sand fly tra gene, as observed in Brachycera and in other insect orders, even though not linked to the presence of the TRACAM domain. The increasing number of released insect genomes/transcriptomes and the availability of the sand fly tra gene sequence will help in future to discover the eventual presence of tra homologs in other Nematocera species, many of them representing important vectors of human diseases. Our model needs to be confirmed by functional analyses and verified also in New World sand fly species, where the tra gene seems to be absent.

A further interesting question to be addressed in future is relative to the molecular nature of the primary signals of sex determination in sand flies, to date completely unknown. To this aim, the $P$. perniciosus species could be an optimal starting point considering that it is the only Old World sand fly species with described heteromorphic sexual chromosomes [80]. The identification of male determining factors and sex-specific genomic loci in sand fly species could not only help to complete the understanding of sex determination mechanisms in Nematocera but also to shed light on chromosome evolution in insects [81-84].

Finally, our results open the possibility of future biotechnological applications to control natural populations of sand flies to reduce their impact on public health by 
using technologies available for other insect pests [85, 86]. In particular, the tra gene could be utilized to produce sexing strains to be implemented for SIT-based control program [85, 87], still missing for sand flies, while the $d s x$ gene could be used to develop gene drive systems for population suppression, as recently proposed for Anopheles mosquitoes [72].

\section{Methods}

\section{Sand flies sex determination genes cloning}

The samples of $P$. perniciosus used in this study were from laboratory colonies held at the PV laboratory (Charles University, Department of Parasitology, Prague - Czech Republic) and at the LG laboratory (Istituto Superiore di Sanità, Rome - Italy). The samples of $P$. papatasi and L. longipalpis used in this study were from laboratory colonies held at the PV laboratory (Charles University, Department of Parasitology, Prague - Czech Republic). The sand fly colonies were reared under standard conditions as previously described [88]. Total RNA was extracted from pools of virgin males and sugar-fed females (7-10 days old) of adult $P$. perniciosus, $P$. papatasi and L. longipalpis using the PureLink ${ }^{\bullet}$ RNA Mini kit (Life Technologies) according to manufacturer's instruction, followed by on-column PureLink ${ }^{\circ}$ DNase (Ambion) treatment. Total RNA was resuspended in $100 \mu \mathrm{l}$ of $\mathrm{ddH}_{2} \mathrm{O}$ and quantified using the NanoDrop 2000c spectrophotometer. The protein coding sequences of insect sex determining genes were used as query to perform TBLASTN search against the PERNI data set (Additional file 1: Table S1) [40]. The transcripts corresponding to the putative $P$. perniciosus orthologues (Additional file 1: Table S1) were utilized to design PCR primer pairs (see Additional file 15: Supplementary Methods). First-strand cDNA was synthesized from 200 ng of male and female total RNA using the EuroScript Reverse Transcriptase kit (Euroclone) with oligo-dT, in a final volume of $20 \mu \mathrm{l}$. To amplify the orthologue of the fruitless gene, cDNA was synthesized with the genespecific primers. PCR amplifications were performed on $1 \mu \mathrm{l}$ of 1:20 dilution of the cDNA template from adult males and females, in a final volume of $50 \mu \mathrm{l}$, using the Dreamtaq DNA polymerase (Thermo Fisher Scientific) or the PfuUltra HF DNA polymerase (Agilent Technologies). Primer melting temperatures $(\mathrm{Tm})$ are reported in Additional file 15: Supplementary Methods. Appropriate annealing temperatures were adjusted to individual primer pairs using in case of different Tm for each primer, the lowest. The 3' end of the Ppetra cDNAs were determined with the 3' RACE System for Rapid Amplification of cDNA Ends (Invitrogen); the 5' end of the Ppetra cDNA was determined with the $5^{\prime} / 3^{\prime}$ RACE kit 2nd generation (Roche). Reverse transcription was performed as recommended by the suppliers. The obtained cDNA fragments were cloned using the Strataclone PCR cloning Kit (Agilent Technologies), and the positive clones were sequenced on an ABI 310 Automated Sequencer (Applied Biosystems). cDNA sequences were deposited at the GenBank database with the following accession numbers: PpedsxM MK286442; PpedsxF MK286443; PpetraM1 MK286444; PpetraM2 MK286445; PpetraF1 MK286446; PpetraF2 MK286447; PpetraF3 MK286448; Ppetra-2A MK286449; Ppetra-2B MK286450; PpefruMA MK286451; PpefruMC MK286452; PpefruFA MK286453; PpefruFC MK286454; PpatraF MK286455.

\section{Developmental expression analysis}

Total RNA was extracted from the different developmental stages of $P$. perniciosus (embryos, 1st, 2nd and 4th instar, and pupae) using the High Pure RNA Tissue Kit (Roche) according to manufacturer's instruction, followed by on-column DNase treatment. First-strand cDNA was synthesized from $0.5 \mu \mathrm{g}$ of total RNA using the First Strand cDNA Synthesis Kit for RT-PCR with both oligo-dT primers and random examers, or with the fruC-nested gene-specific primer. PCR amplifications were performed on $1 \mu \mathrm{l}$ of 1:20 dilution of the cDNA template in a final volume of $50 \mu \mathrm{l}$ using the EmeraldAmp PCR Master Mix (Clontech). Appropriate annealing temperatures and cycle conditions were adjusted to individual primer pairs (see Additional file 15 Supplementary Methods).

\section{Ppetra genomic organization}

To identify the intronic region sequence of the $P$. perniciosus transformer gene, genomic DNA was extracted from a single adult female using the NucleoSpin Tissue XS (Macherey-Nagel) according to manufacturer. PCR amplification was conducted on $10 \mathrm{ng}$ of genomic DNA in a final volume of $50 \mu \mathrm{l}$ using the primers Ppetra5utr/ Ppetrastop3utr and the following thermal cycle: $95^{\circ} \mathrm{C}$ for $3 \mathrm{~min}, 35 \mathrm{cycles}$ of $94^{\circ} \mathrm{C}$ for $30 \mathrm{~s}, 56^{\circ} \mathrm{C}$ for $30 \mathrm{~s}, 72^{\circ} \mathrm{C}$ for $2.30 \mathrm{~min}$, final extension of $10 \mathrm{~min}$ at $72^{\circ} \mathrm{C}$. The amplification product was cloned and sequenced as described above. The Ppetra genomic locus sequence was deposited at the GenBank database with the accession number MK286466.

\section{Phylogeny and evolutionary analysis}

Nucleotide and encoded amino acid sequence of homologs of the Ppedsx, Ppefru, Ppetra and Ppetra-2 genes were downloaded from GenBank and the relative accession numbers are listed in Table S3. Amino acid sequences were aligned using MUSCLE. Due to high sequence divergence, for each gene the alignments were restricted to the encoded protein regions whose alignment is not ambiguous, as follow: TRA (TRACAM domain and DIPTERA domain), TRA-2 (RRM domain and 
linker region), DSXF (OD1 and OD2 domains), FRUM (Male-specific $\mathrm{N}$-terminal region and BTB domain). Based on their amino acid alignments, nucleotide sequences were aligned using PAL2NAL. Maximum Likelihood trees were constructed on the nucleotide alignments using MEGA7 [89], with 1000 bootstrap replicates. For each gene, the best evolutionary model was selected using MEGA7: Kimura 2-parameter for tra $(\mathrm{K} 2+\mathrm{G})$ and for tra-2 $(\mathrm{K} 2+\mathrm{G}+\mathrm{I})$, General Time Reversible for $d s x(\mathrm{GTR}+\mathrm{G})$ and Hasegawa-Kishino-Yano for fru $(\mathrm{HKY}+\mathrm{G})$. The coding sequences of the $d s x$, fru, tra and tra-2 homolog genes were analyzed with the CODEML program from PAML v.4.8 [90] to evaluate their evolutionary rates. Different evolutionary models were compared (branch, sites and branch-sites) to test for variation of the ratio between nonsynonymous and synonymous substitution rate $(\omega)$ at specific codons in the sequences and among the branches of the trees. For each comparison, a likelihood ratio test was applied to establish which model best fits the data.

\section{Additional files}

Additional file 1: Table S1. TBLASTN search of sex determination orthologs in the perniBASE dataset. (XLS $11 \mathrm{~kb}$ )

Additional file 2: Figure S1. Multiple sequence alignment of SXL proteins. Figure S2. Multiple sequence alignment of TRA-2 proteins. Figure S3. Multiple sequence alignment of DSX amino-terminal regions. Figure S4. Multiple sequence alignment of DSXF carboxy-terminal regions. Figure S5. Multiple sequence alignment of DSXM carboxyterminal regions. Figure S6. Multiple sequence alignment of FRUM amino-terminal regions. Figure S7. Multiple sequence alignment of FRU proteins. Figure S8. Sex-lethal gene expression at adult stage in $P$. perniciosus. Figure S9. Multiple sequence alignment of TRA proteins. (PDF $895 \mathrm{~kb}$ )

Additional file 3: Figure S10. Manually-curated P. perniciosus transformer gene model. Figure S11. Manually-curated $P$. papatasi transformer gene model. Figure S12. Manually-curated $P$. bergeroti transformer gene model. Figure S13. Manually-curated P. duboscai transformer gene model. (PDF 349 $\mathrm{kb})$

Additional file 4: Figure S14. Multiple sequence alignment of TRA proteins in Phlebotomus spp. (PDF $251 \mathrm{~kb}$ )

Additional file 5: Figure S15. Multiple alignment of tra introns in Phlebotomus spp. (PDF $572 \mathrm{~kb}$ )

Additional file $\mathbf{6}$ : Figure $\mathbf{S 1 6}$. tra gene expression at adult stage in $P$. papatasi. (PDF $137 \mathrm{~kb}$ )

Additional file 7: Figure S17. Manually-curated P. papatasi tra-2 partial gene model. Figure S18. Manually-curated $P$. bergeroti tra-2 partial gene model. Figure S19. Manually-curated P. duboscai tra-2 partial gene model. (PDF $142 \mathrm{~kb}$ )

Additional file 8: Figure S20. Multiple sequence alignment of sand fly TRA-2 proteins. (PDF $93 \mathrm{~kb}$ )

Additional file 9: Figure S21. Manually-curated $P$. papatasi doublesex gene model. Figure S22. Manually-curated L. longipalpis doublesex gene model. Figure S23. Manually-curated P. papatasi fruitless gene model. Figure S24. Manually-curated L. longipalpis fruitless partial gene model. Figure S25. Comparison of genomic structures of dipteran $d s x$ genes. Figure S26. Comparison of genomic structures of dipteran fru genes. (PDF $283 \mathrm{~kb}$ )
Additional file 10: Figure S27. Crispr/Cas9 target sites in sand fly $d s x$ genes. (PDF $155 \mathrm{~kb}$ )

Additional file 11: Table S2. TRA/TRA-2 binding sites of Brachycera and Nematocera species. (XLS $16 \mathrm{~kb}$ )

Additional file 12: Figure S28. Manually-curated $P$. bergeroti fruitless partial gene model. Figure $\mathbf{S 2 9}$. Manually-curated $P$. duboscai fruitless partial gene model. Figure S30. Manually-curated $P$. bergeroti doublesex partial gene model. Figure S31. Manually-curated P. duboscai doublesex partial gene model. (PDF $142 \mathrm{~kb}$ )

Additional file 13: Table S3. Accession numbers and ID of the sequences used in phylogenetic and evolutionary analyses. (XLS $15 \mathrm{~kb}$ )

Additional file 14: Table S4. Statistics of the evolutionary analyses and comparison of different evolutionary models. (XLS $16 \mathrm{~kb}$ )

Additional file 15: Supplementary Methods. (PDF 345 kb)

\section{Abbreviations}

BTB: Broad-Complex, Tramtrack and Bric a brac; DM: Doublesex Mab3; ORF: Open Reading Frame; RACE: Rapid Amplification of CDNA END:; RRM: RNA Recognition Motif; TRACAM: TRA Ceratitis-Apis-Musca

\section{Acknowledgements}

The authors are deeply grateful to Riccardo Bianchi and Marta Marchili, Istituto Superiore di Sanità, Roma, Italy, for technical support in rearing sand flies. We deeply acknowledge the Sand Fly Genome Sequencing Consortium and the Baylor College of Medicine Human Genome Sequencing Center (BCM-HGSC) for releasing their unpublished data prior to project completion.

\section{Authors' contributions}

MS conceived the study. MS and VP planned the experiments. VP performed all the molecular analyses. NP helped with DNA and RNA extractions and RT-PCR analyses. MS performed all bioinformatic analysis with additional contribution of SA, VC and RS. GS suggested the search for tra ortholog by using TRATTRA-2 binding site sequences. MS performed the manual curation of sex determination genes and comparative genomics analyses. SA performed the phylogeny and evolutionary analyses. PV contributed with reagents and biological samples, GB and LG maintained the $P$. perniciosus colony and collected samples. MS, SA and VC wrote the manuscript with inputs by GS, RS, PV and LG. All authors read and approved the final manuscript.

\section{Funding}

This study was supported the grant STAR2013_25 to MS from University of Naples Federico II and Compagnia di San Paolo, Naples, ITALY, in the frame of Programme STAR2013 (Sostegno Territoriale alle Attività di Ricerca).

\section{Availability of data and materials}

All the sequencing data produced in this work are deposited in the GenBank public database or present in the Additional file 15 Supplementary Methods. P. perniciosus transcriptome assembly utilized in this work is freely available at http://pernibase.evosexdevo.eu and the corresponding RNA-seq raw data are available at the SRA NCBI database under the accession number PRJNA287743. Genome or transcriptome assemblies produced in this study are available upon request or reproducible using instructions present in the Additional file 15 Supplementary Methods.

Ethics approval and consent to participate

Not applicable.

\section{Consent for publication}

Not applicable.

\section{Competing interests}

The authors declare that they have no competing interests.

\section{Author details}

'Department of Biology, University of Naples Federico II, Naples, Italy. ${ }^{2}$ National Research Council, Institute of Genetics and Biophysics, Naples, Italy. ${ }^{3}$ Stazione Zoologica "Anton Dohrn", Naples, Italy. ${ }^{4}$ Department of Neuroscience, Scuola Internazionale Superiore di Studi Avanzati (SISSA), Trieste, Italy. ${ }^{5}$ Department of Parasitology, Charles University, Prague, Czech 
Republic. ${ }^{6}$ Unit of Vector-borne Diseases, Department of Infectious Diseases, Istituto Superiore di Sanità, Rome, Italy.

\section{Received: 25 January 2019 Accepted: 11 June 2019 Published online: 25 June 2019}

\section{References}

1. Haag ES, Doty AV. Sex determination across evolution: Connecting the dots. PLoS Biol. 2005;3(1):e21.

2. Zarkower D. Establishing sexual dimorphism: conservation amidst diversity? Nat Rev Genet. 2001;2:175-85.

3. Marshall Graves JA. Weird animal genomes and the evolution of vertebrate sex and sex chromosomes. Annu Rev Genet. 2008:42:565-86.

4. Verhulst EC, Van De Zande L. Insect sex determination: a cascade of mechanisms. Sex Dev. 2014;8:5-6.

5. Sánchez L. Sex-determining mechanisms in insects. Int J Dev Biol. 2008;52: 837-56.

6. Gempe T, Beye M. Function and evolution of sex determination mechanisms, genes and pathways in insects. BioEssays. 2011;33:52-60

7. Schütt C, Nöthiger R. Structure, function and evolution of sex-determining systems in dipteran insects. Development. 2000;127:667-77.

8. Erickson JW, Quintero JJ. Indirect effects of ploidy suggest X chromosome dose, not the X:a ratio, signals sex in Drosophila. PLoS Biol. 2007;5:2821-30

9. Saccone G, Pane A, Polito LC. Sex determination in flies, fruitflies and butterflies. Genetica. 2002;116:15-23.

10. Bopp D, Saccone G, Beye M. Sex determination in insects: variations on a common theme. Sex Dev. 2014;8:20-8.

11. Sharma A, Heinze SD, Wu Y, Kohlbrenner T, Morilla I, Brunner C, et al. Male sex in houseflies is determined by Mdmd, a paralog of the generic splice factor gene CWC22. Science. 2017;356:642-5.

12. Pane A, Salvemini M, Delli Bovi P, Polito C, Saccone G. The transformer gene in Ceratitis capitata provides a genetic basis for selecting and remembering the sexual fate. Development. 2002;129:3715-25.

13. Salvemini M, Robertson M, Aronson B, Atkinson P, Polito LC, Saccone G. Ceratitis capitata transformer-2 gene is required to establish and maintain the autoregulation of Cctra, the master gene for female sex determination. Int J Dev Biol. 2009:53:109-20.

14. Verhulst EC, van de Zande L, Beukeboom LW. Insect sex determination: it all evolves around transformer. Curr Opin Genet Dev. 2010;20:376-83.

15. Katsuma S, Kiuchi T, Kawamoto M, Fujimoto T, Sahara K. Unique sex determination system in the silkworm, Bombyx mori: current status and beyond. Proc Jpn Acad Ser B. 2018;94:205.

16. Saccone G, Salvemini M, Polito LC. The transformer gene of Ceratitis capitata: a paradigm for a conserved epigenetic master regulator of sex determination in insects. Genetica. 2011;139:99-111.

17. Ruiz MF, Milano A, Salvemini M, Eirín-López JM, Perondini ALP, Selivon D, et al. The gene transformer of anastrepha fruit flies (Diptera, tephritidae) and its evolution in insects. PLoS One. 2007;2:e1239.

18. Li F, Vensko SP, Belikoff EJ, Scott MJ. Conservation and sex-specific splicing of the transformer gene in the Calliphorids Cochliomyia hominivorax, Cochliomyia macellaria and Lucilia sericata. PLoS One. 2013;8:1-14.

19. Hediger M, Henggeler C, Meier N, Perez R, Saccone G, Bopp D. Molecular characterization of the key switch $\mathrm{F}$ provides a basis for understanding the rapid divergence of the sex-determining pathway in the housefly. Genetics. 2010;184:155-70.

20. Concha C, Scott MJ. Sexual development in Lucilia cuprina (Diptera, Calliphoridae) is controlled by the transformer gene. Genetics. 2009;182: 785-98.

21. Luo Y, Zhao S, Li J, Li P, Yan R. Isolation and molecular characterization of the transformer gene from Bactrocera cucurbitae (Diptera: Tephritidae). J Insect Sci. 2017;17(2).

22. Laohakieat $\mathrm{K}$, Aketarawong $\mathrm{N}$, Isasawin S, Thitamadee S, Thanaphum S. The study of the transformer gene from Bactrocera dorsalis and B. correcta with putative core promoter regions. BMC Genet. 2016;17:34.

23. Salvemini M, D'Amato R, Petrella V, Aceto S, Nimmo D, Neira M, et al. The orthologue of the fruitfly sex behaviour gene fruitless in the mosquito Aedes aegypti: evolution of genomic organisation and alternative splicing. PLoS One. 2013;8:e48554.

24. Salvemini M, Mauro U, Lombardo F, Milano A, Zazzaro V, Arcà B, et al. Genomic organization and splicing evolution of the doublesex gene, a
Drosophila regulator of sexual differentiation, in the dengue and yellow fever mosquito Aedes aegypti. BMC Evol Biol. 2011;11:41.

25. Gailey DA, Billeter JC, Liu JH, Bauzon F, Allendorfer JB, Goodwin SF. Functional conservation of the fruitless male sex-determination gene across 250 Myr of insect evolution. Mol Biol Evol. 2006;23:633-43.

26. Scali C. Identification of sex-specific transcripts of the Anopheles gambiae doublesex gene. J Exp Biol. 2005;208:3701-9.

27. Criscione F, Qi Y, Tu Z. GUY1 confers complete female lethality and is a strong candidate for a male-determining factor in anopheles Stephensi. Elife. 2016;5.

28. Krzywinska E, Dennison NJ, Lycett GJ, Krzywinski J. A maleness gene in the malaria mosquito Anopheles gambiae. Science. 2016;353:67-9.

29. Hall AB, Basu S, Jiang X, Qi Y, Timoshevskiy VA, Biedler JK, et al. A maledetermining factor in the mosquito Aedes aegypti. Science. 2015;348:1268-70.

30. Biedler JK, Tu Z. Sex determination in mosquitoes. In: Advances in Insect Physiology; 2016. p. 37-66

31. Maroli M, Feliciangeli MD, Bichaud L, Charrel RN, Gradoni L. Phlebotomine sandflies and the spreading of leishmaniases and other diseases of public health concern. Med Vet Entomol. 2013;27:123-47.

32. Young DG, Duncan MA. Guide of the identification and geografic distribution of Lutzomyia sand flies in Mexico, West Indies, central and South America (Diptera: Psychodidae). Mem Am Entomol Inst. 1994;54: $1-881$.

33. WHO. Control of the Leishmaniases. Geneva: World Health Organization; 2010.

34. Alvar J, Vélez ID, Bern C, Herrero M, Desjeux P, Cano J, et al. Leishmaniasis worldwide and global estimates of its incidence. PLoS One. 2012;7(5):e35671.

35. Depaquit J, Grandadam M, Fouque F, Andry PE, Peyrefitte C. Arthropodborne viruses transmitted by Phlebotomine sandflies in Europe: a review. Euro Surveill. 2010;15:19507.

36. Volff JN, Zarkower D, Bardwell VJ, Schartl M. Evolutionary dynamics of the DM domain gene family in metazoans. J Mol Evol. 2003;57 Suppl 1:S241-9.

37. Perez-Torrado R, Yamada D, Defossez PA. Born to bind: the BTB proteinprotein interaction domain. BioEssays. 2006;28:1194-202.

38. Cléry A, Blatter M, Allain FHT. RNA recognition motifs: boring? Not quite. Curr Opin Struct Biol. 2008;18:290-8.

39. Geuverink E, Beukeboom LW. Phylogenetic distribution and evolutionary dynamics of the sex determination genes doublesex and transformer in insects. Sex Dev. 2014;8:38-49.

40. Petrella V, Aceto S, Musacchia F, Colonna V, Robinson M, Benes V, et al. De novo assembly and sex-specific transcriptome profiling in the sand fly Phlebotomus perniciosus (Diptera, Phlebotominae), a major Old World vector of Leishmania infantum. BMC Genomics. 2015;16:847.

41. McAllister BF, McVean GAT. Neutral evolution of the sex-determining gene transformer in Drosophila. Genetics. 2000;154:1711-20.

42. Kulathinal RJ, Skwarek L, Morton RA, Singh RS. Rapid evolution of the sexdetermining gene, transformer: structural diversity and rate heterogeneity among sibling species of drosophila. Mol Biol Evol. 2003;20:441-52.

43. Serna E, Gorab E, Ruiz MF, Goday C, Eirín-López JM, Sánchez L. The gene sex-lethal of the sciaridae family (order diptera, suborder nematocera) and its phylogeny in dipteran insects. Genetics. 2004;168:907-21.

44. Traut W, Niimi T, Ikeo K, Sahara K. Phylogeny of the sex-determining gene Sex-lethal in insects. Genome. 2006;49:254-62.

45. Meise M, Hilfiker-Kleiner D, Dübendorfer A, Brunner C, Nöthiger R, Bopp D. Sex-lethal, the master sex-determining gene in Drosophila, is not sex-specifically regulated in Musca domestica. Development. 1998;125: 1487-94.

46. Saccone G, Peluso I, Artiaco D, Giordano E, Bopp D, Polito LC. The Ceratitis capitata homologue of the Drosophila sex-determining gene sex-lethal is structurally conserved, but not sex-specifically regulated. Development. 1998;125:1495-500,

47. Graham P, Penn JKM, Schedl P. Masters change, slaves remain. BioEssays. 2003:25:1-4

48. Xie W, Guo L, Jiao X, Yang N, Yang X, Wu Q, et al. Transcriptomic dissection of sexual differences in Bemisia tabaci, an invasive agricultural pest worldwide. Sci Rep. 2014;4:4088.

49. Hedley ML, Maniatis T. Sex-specific splicing and polyadenylation of dsx premRNA requires a sequence that binds specifically to tra- 2 protein in vitro. Cell. 1991;65:579-86.

50. Baker BS, Heinrichs V, Ryner LC. Regulation of sex-specific selection of fruitless $5^{\prime}$ splice sites by transformer and transformer-2. Mol Cell Biol. 1998; $18: 450-8$. 
51. Burghardt $G$, Hediger M, Siegenthaler C, Moser M, Dübendorfer A, Bopp D. The transformer2 gene in Musca domestica is required for selecting and maintaining the female pathway of development. Dev Genes Evol. 2005; 215:165-76.

52. Burtis KC, Baker BS. Drosophila doublesex gene controls somatic sexual differentiation by producing alternatively spliced mRNAs encoding related sex-specific polypeptides. Cell. 1989;56:997-1010.

53. Saccone G, Salvemini M, Pane A, Polito LC. Masculinization of XX Drosophila transgenic flies expressing the Ceratitis capitata DoublesexM isoform. Int J Dev Biol. 2008;52:1051-7.

54. Salvemini M, Polito C, Saccone G. Fruitless alternative splicing and sex behaviour in insects: an ancient and unforgettable love story? J Genet. 2010;89:287-99.

55. Salikhov K, Sacomoto G, Kucherov G. Using cascading bloom filters to improve the memory usage for de Brujin graphs. In: Lecture Notes in Computer Science (including subseries Lecture Notes in Artificial Intelligence and Lecture Notes in Bioinformatics); 2013. p. 364-76.

56. Grabherr MG, Haas BJ, Yassour M, Levin JZ, Thompson DA, Amit I, et al. Fulllength transcriptome assembly from RNA-Seq data without a reference genome. Nat Biotechnol. 2011;29:644-52.

57. Haas BJ, Papanicolaou A, Yassour M, Grabherr M, Philip D, Bowden J, et al. Reference generation and analysis with trinity. Nat Protoc. 2014;8:1-43.

58. Bolger AM, Lohse M, Usadel B. Trimmomatic: a flexible trimmer for Illumina sequence data. Bioinformatics. 2014;30:2114-20.

59. Mita K, Kasahara M, Sasaki S, Nagayasu Y, Yamada T, Kanamori H, et al. The genome sequence of silkworm, Bombyx mori. DNA Res. 2004;11:27-35.

60. Akhoundi M, Kuhls K, Cannet A, Votýpka J, Marty P, Delaunay P, et al. A historical overview of the classification, evolution, and dispersion of Leishmania parasites and sandflies. PLoS Negl Trop Dis. 2016;10(3):e0004349.

61. Belote JM, Baker BS. Sex determination in Drosophila melanogaster: analysis of transformer-2, a sex-transforming locus. Proc Natl Acad Sci U S A. 1982; 79:1568-72.

62. Goralski TJ, Edström JE, Baker BS. The sex determination locus transformer-2 of Drosophila encodes a polypeptide with similarity to RNA binding proteins. Cell. 1989;56:1011-8.

63. Chandler D, McGuffin ME, Piskur J, Yao J, Baker BS, Mattox W. Evolutionary conservation of regulatory strategies for the sex determination factor transformer-2. Mol Cell Biol. 1997:17:2908-19.

64. Gomulski LM, Dimopoulos G, Xi Z, Soares MB, Bonaldo MF, Malacrida AR, et al. Gene discovery in an invasive tephritid model pest species, the Mediterranean fruit fly, Ceratitis capitata. BMC Genomics. 2008;9:243.

65. Sarno F, Ruiz MF, Eirín-López JM, Perondini AL, Selivon D, Sánchez L. The gene transformer-2 of Anastrepha fruit flies (Diptera, Tephritidae) and its evolution in insects. BMC Evol Biol. 2010;10:140.

66. Martín I, Ruiz MF, Sánchez L. The gene transformer-2 of Sciara (Diptera, Nematocera) and its effect on Drosophila sexual development. BMC Dev Biol. 2011:11:19.

67. Dauwalder B, Amaya-Manzanares F, Mattox W. A human homologue of the Drosophila sex determination factor transformer-2 has conserved splicing regulatory functions. Proc Natl Acad Sci. 1996;93:9004-9.

68. Liu G, Wu Q, Li J, Zhang G, Wan F. RNAi-mediated knock-down of transformer and transformer 2 to generate male-only progeny in the oriental fruit fly, Bactrocera dorsalis (Hendel). PLoS One. 2015;10(6):e0128892.

69. Hoang KP, Teo TM, Ho TX, Le VS. Mechanisms of sex determination and transmission ratio distortion in Aedes aegypti. Parasit Vectors. 2016;9:49.

70. Hediger M, Burghardt G, Siegenthaler C, Buser N, Hilfiker-Kleiner D, Dübendorfer $\mathrm{A}$, et al. Sex determination in Drosophila melanogaster and Musca domestica converges at the level of the terminal regulator doublesex. Dev Genes Evol. 2004;214:29-42.

71. Kuhn S, Sievert V, Traut W. The sex-determining gene doublesex in the fly Megaselia scalaris: conserved structure and sex-specific splicing. Genome. 2000:43:1011-20.

72. Kyrou K, Hammond AM, Galizi R, Kranjc N, Burt A, Beaghton AK, et al. A CRISPR-Cas9 gene drive targeting doublesex causes complete population suppression in caged Anopheles gambiae mosquitoes. Nat Biotechnol. 2018;36:1062-6.

73. Ryner LC, Baker BS. Regulation of doublesex pre-mRNA processing occurs by 3'-splice site activation. Genes Dev. 1991;5:2071-85.

74. Hoshijima K, Inoue K, Higuchi I, Sakamoto H, Shimura Y. Control of doublesex alternative splicing by transformer and transformer-2 in Drosophila. Science. 1991;252:833-6.
75. Ruiz MF, Alvarez M, Eirín-López JM, Sarno F, Kremer L, Barbero JL, et al. An unusual role for doublesex in sex determination in the dipteran Sciara. Genetics. 2015;200:1181-99.

76. Price DC, Egizi A, Fonseca DM. Characterization of the doublesex gene within the Culex pipiens complex suggests regulatory plasticity at the base of the mosquito sex determination cascade. BMC Evol Biol. 2015;15:108.

77. Yang Z, Nielsen R. Synonymous and nonsynonymous rate variation in nuclear genes of mammals. J Mol Evol. 1998;46:409-18.

78. Yang Z, Wong WSW, Nielsen R. Bayes empirical Bayes inference of amino acid sites under positive selection. Mol Biol Evol. 2005;22:1107-18.

79. Zhang J, Nielsen R, Yang Z. Evaluation of an improved branch-site likelihood method for detecting positive selection at the molecular level. Mol Biol Evol. 2005;22:2472-9.

80. Kreutzer RD, Modi GB, Tesh RB, Young DG. Brain cell karyotypes of six species of new and Old World sand flies (Diptera: Psychodidae). J Med Entomol. 1987;24:609-12.

81. Cortez D, Marin R, Toledo-Flores D, Froidevaux L, Liechti A, Waters PD, et al. Origins and functional evolution of $Y$ chromosomes across mammals. Nature. 2014;508:488-93.

82. Blackmon H, Ross L, Bachtrog D. Sex Determination, Sex Chromosomes, and Karyotype Evolution in Insects. J Hered. 2016:esw047.

83. Kaiser VB, Bachtrog D. Evolution of sex chromosomes in insects. Annu Rev Genet. 2010;44:91-112

84. Vyskot B. Y Chromosome Evolution. In: Brenner's Encyclopedia of Genetics. 2nd ed; 2013. p. 372-5.

85. Papathanos PA, Bossin HC, Benedict MQ, Catteruccia F, Malcolm CA, Alphey $L$, et al. Sex separation strategies: past experience and new approaches. Malar J. 2009;8(Suppl 2):S5

86. Hendrichs J, Robinson A. Sterile Insect Technique. In: Encyclopedia of Insects; 2009. p. 953-7.

87. Saccone G, Pane A, De Simone A, Salvemini M, Milano A, Annunziata L, et al. New Sexing Strains for Mediterranean Fruit Fly Ceratitis capitata: Transforming Females into Males. In: Vreysen M.J.B., Robinson A.S. Hendrichs J. (eds) Area-Wide Control of Insect Pests. Dordrecht: Springer; 2007. p. 95-102.

88. Volf $\mathrm{P}$, Volfova $\mathrm{V}$. Establishment and maintenance of sand fly colonies. J Vector Ecol. 2011:36 Suppl 1:S1-9.

89. Sudhir Kumar, Glen Stecher, Koichiro Tamura, (2016) MEGA7: Molecular Evolutionary Genetics Analysis Version 7.0 for Bigger Datasets. Molecular Biology and Evolution 33 (7):1870-1874

90. Yang Z. PAML 4: phylogenetic analysis by maximum likelihood. Mol Biol Evol. 2007; 24(8):1586-91.

\section{Publisher's Note}

Springer Nature remains neutral with regard to jurisdictional claims in published maps and institutional affiliations.
Ready to submit your research? Choose BMC and benefit from:

- fast, convenient online submission

- thorough peer review by experienced researchers in your field

- rapid publication on acceptance

- support for research data, including large and complex data types

- gold Open Access which fosters wider collaboration and increased citations

- maximum visibility for your research: over $100 \mathrm{M}$ website views per year

At $\mathrm{BMC}$, research is always in progress.

Learn more biomedcentral.com/submissions 